\title{
An Application of a Delay CSOH Model on the Coconut Farm
}

\author{
Adirek Vajrapatkul, Sanoe Koonprasert, Sekson Sirisubtawee
}

\begin{abstract}
In this paper, we introduce the mathematical model that represents the quantity and population dynamics on the coconut farm. The model encompasses the number of coconuts and population of squirrels, barn owls, and squirrel hunters. We study the fundamental properties of the model that include positivity, boundedness, and equilibrium points. We also investigate the effect of the time delay on the stability of the equilibrium points. The results of the analysis show that when the time delay reaches its critical value, the interior equilibrium point lost its stability, and there occurs the Hopf bifurcation.
\end{abstract}

Index Terms: Samut Songkhram, Thailand, squirrels, coconuts, bran owls, squirrel hunters, $\mathrm{CSOH}$ model, time delay.

\section{INTRODUCTION}

Samut Songkhram, a province of Thailand, where coconut trees are grown extensively, has encountered the problem of squirrels eating the coconuts of farmers. The invasion of squirrels leads to a significant reduction of coconut yields and income of the farmers. Although the coconut farmers can prevent their coconuts from squirrels by trapping and hunting practice, they still searching for alternative practices to control squirrels to a particular density that allow them to have more stable or predictable coconut outputs and income. To identify controlling strategies, mathematical modeling becomes a popular technique. The models that are constructed and studied are often based on the concept of prey-predator relationship proposed in 1931 by Lotka-Volterra [1] . The works in this area could be found in, e.g., [2]-[6] . These kinds of the model are studied and investigate for the stability of the solutions and also the conditions for bifurcation. This bifurcation can be emerged by various parameters. However, the time delay is a parameter that is intensively studied for the bifurcation phenomenon purpose. The works that were conducted to investigate the effect of the time delay on the stability of solution could be found in, e.g., [7] which analyze the effect of time delay in the following model:

\footnotetext{
Revised Manuscript Received on September 22, 2019.

Adirek Vajrapatkul is a Ph.D. student in the Department of Mathematics, King Mongkut's University of Technology North Bangkok, 10800 Thailand.

Sekson Sirisubtawee is a lecturer in the Department of Mathematics, King Mongkut's University of Technology North Bangkok, 10800 Thailand, and a researcher in the Centre of Excellence in Mathematics, CHE, Si Ayutthaya Road, Bangkok 10400, Thailand.

Sanoe Khoonprasert is an Associate Professor in the Department of Mathematics, King Mongkut's University of Technology North Bangkok, 10800 Thailand, and a researcher in the Centre of Excellence in Mathematics, CHE, Si Ayutthaya Road, Bangkok 10400, Thailand.
}

$$
\begin{aligned}
& \frac{d S}{d t}=r S\left(1-\frac{S+I}{k}\right)-\theta I S, \\
& \frac{d I}{d t}=\theta I S-\mu I-m I y_{m}, \\
& \frac{d y_{i}}{d t}=\alpha y_{m}-\alpha y_{m}(t-\tau) e^{-r \tau}-\gamma y_{i}, \\
& \frac{d y_{m}}{d t}=\alpha y_{m}(t-\tau) e^{-r \tau}-\beta y_{m}^{2}+\pi m y_{m},
\end{aligned}
$$

where $S(t)$ and $I(t)$ denote the population of susceptible prey and infected prey respectively. $y_{i}(t)$ is immature predator population and $y_{m}(t)$ represents mature predator population. Here a constant $\tau$ represent the time delay in transferring to a new stage. An application of time delay to explain the effect of gestation time delay can be found in [8] which integrates the time delay in the following model:

$$
\begin{aligned}
& \frac{d N(t)}{d t}=\left(a+\xi_{1}(t)-b N(t)\right) N(t)-\frac{c N(t) P(t)}{m P(t)+1}, \\
& \frac{d P(t)}{d t}=\left(-d+\xi_{2}(t)+\frac{k N(t-\tau)}{m P(t-\tau)+1}\right) P(t),
\end{aligned}
$$

where $N(t)$ and $P(t)$ describe the prey and predator population. $\tau$ is the gestation period or reaction time of the predators. Alternatively, time delay in convert food to the growth rate can be found in [9] which present the time delay in the following model:

$$
\begin{aligned}
& \frac{d N(t)}{d T}=R N(t)\left(1-\frac{N(t-\tau)}{K}\right)-\frac{C N(t) P(t)}{A+N(t)}-\frac{F E N}{m_{1} E+m_{2} N}, \\
& \frac{d P(t)}{d T}=\frac{C_{1} N(t) P(t)}{A_{1}+N(t)}-D_{1} P(t)-\frac{C_{2} P(t) Q(t)}{A_{2}+P(t)+B_{1} Q(t)} \\
& \frac{d Q(t)}{d T}=D_{2} Q(t)^{2}-\frac{C_{3} Q(t)^{2}}{A_{3}+P(t)}
\end{aligned}
$$

where $N(t), P(t)$ and $Q(t)$ are the prey, middle-level predator and top-level predator population respectively. Here $\tau$ denote time delay to convert the food into growth of the pray. In addition, the model that consists of two time delays could be found in [10] which study the effect of time delay in the following model:

$$
\begin{aligned}
& \frac{d x}{d t}=x\left(\eta-b_{1} x\left(t-\tau_{1}\right)\right)-\frac{a_{1} x y}{x+k_{1}}, \\
& \frac{d y}{d t}=y\left(\eta_{2}-\frac{a_{2} y\left(t-\tau_{2}\right)}{x\left(t-\tau_{2}\right)+k_{2}}\right),
\end{aligned}
$$


where $x(t)$ and $y(t)$ denote the population densities of the prey and the predator. $\tau_{1}$ is the feedback delay of the prey to the growth of the species itself. $\tau_{2}$ is the feedback delay of the predator.

Other applications of two time delay model could be found in, e.g., [11]-[14].

Motivate by the squirrel situation on the coconut farm in Samut Songkhram and the effect of the time delay on the stability of solutions, we thus propose the delay CSOH model to explain the effect of time delay on the stability of the squirrel density and especially the coconut outputs. This paper is organized as follows. In Section 2, we explain our model. We analyze the basic properties of the model in Section 3 and Section 4. The conditions for stability and Hopf bifurcation are derived in Section 5. Finally, the numerical simulations and policy discussion are conducted and discussed in Section 6.

\section{CSOH MODEL FORMULATION}

The interaction between coconut yields, squirrels, barn owls, and squirrel hunters within the coconut farm can be represented as follow:

$$
\begin{aligned}
& \frac{d C(t)}{d t}= \beta C(t)\left(1-\frac{C(t)}{K}\right)-\gamma C(t) S(t)-\mu C(t), \\
& \frac{d S(t)}{d t}=\eta S(t)\left(1-\frac{S(t)}{\omega C(t)+Q}\right)+\lambda C\left(t-\tau_{1}\right) S(t) \\
&- \delta S(t) O(t)-\sigma S(t) H(t), \\
& \frac{d O(t)}{d t}= \xi O(t)\left(1-\frac{O(t)}{\kappa S(t)+B}\right)+\zeta S\left(t-\tau_{2}\right) O(t), \\
& \frac{d H(t)}{d t}= c S(t)-d H(t),
\end{aligned}
$$

with the following history

$[C(t), S(t), O(t), H(t)]^{T}=[C(t), S(t), O(0), H(0)]^{T}$

, $\max \left\{\tau_{1}, \tau_{2}\right\} \leq t \leq 0$.

where $C(t), S(t), O(t)$ and $H(t)$ denote respectively, the number of coconut yields, the densities of squirrels, barn owls, and squirrel hunters at time $t . \beta, \gamma, \mu, \eta, \omega, \lambda$, $\delta, \sigma, \xi, \kappa, \zeta, c$, and $d$ are positive constants. $\tau_{1}$ and $\tau_{2}$ are non-negative constants which represent the time delay of coconut convert to new squirrel biomass and time delay of squirrel convert to new barn owls biomass respectively.

\section{NON-NEGATIVITY AND BOUNDEDNESS OF SOLUTIONS}

The following theorems ensure the positivity and boundedness of system (1).

Theorem: All solutions of (1) with positive initial conditions are positive and ultimately bounded for $\forall t \geq 0$.

Proof: [15], [16] From the first equation of (1), it provides $\frac{d C(t)}{C(t)}=\left(\beta\left(1-\frac{C(t)}{K}\right)-\gamma S-\mu\right) d t$,

which gives
$C(t)=C(0) e^{\int_{0}^{t}\left(\beta\left(1-\frac{C(s)}{K}\right)-\gamma S(s)-\mu\right) d s}>0$.

Similarly, from the second and third equation of (1) we can obtain

$$
\begin{gathered}
S(t)=S(0) e^{\int_{0}^{t}\left(\eta\left(1-\frac{S(s)}{C(s) \omega+Q}\right)+\lambda C(s-\tau)-\delta O(s)-\sigma\right) d s}>0, \\
O(t)=O(0) e^{\int_{0}^{t}\left(\xi\left(1-\frac{O(s)}{S(s) \kappa+B}\right)+\zeta S\left(s-\tau_{2}\right)\right) d s}>0 .
\end{gathered}
$$

Therefore $C(t)>0, S(t)>0$, and $O(t)>0, \forall t \in[0, \infty)$.

To prove $H(t)>0$ on $[0, \infty)$, suppose that there exists $t_{H}>0$ such that $H\left(t_{H}\right)=0, S\left(t_{H}\right) \geq 0$ and $\frac{d}{d t} H\left(t_{H}\right)<0$. From the fourth equation of (1) we have.

$\frac{d}{d t} H\left(t_{H}\right)=c S\left(t_{H}\right)-d H\left(t_{H}\right)$,

that provides

$\frac{d}{d t} H\left(t_{H}\right)=c S\left(t_{H}\right)>0$,

which is contradicted.Therefore $H(t)>0, \forall t \in[0, \infty)$.

Now, we show that all solutions of (1) are uniformly ultimately bounded. From the first equation of (1), it provides $\frac{d}{d t} C(t) \leq \beta C(t)\left(1-\frac{C(t)}{K}\right)-\mu C(t)$,

which gives

$C(t) \leq \max \left\{\frac{(\beta-\mu) K}{\beta}, C_{0}\right\}:=M_{1}$,

and

$\frac{d}{d t} C(t) \leq \beta M_{1}$.

Also applying this process to second equation of (1), we have

$\frac{d}{d t} S(t) \leq \eta S(t)\left(1-\frac{S(t)}{C \omega+Q}\right)+\lambda C\left(t-\tau_{1}\right) S(t)$,

$S(t) \leq \max \left\{\frac{M_{1}^{2} \lambda \omega+(Q \lambda+\eta \omega) M_{l}+Q \eta}{\eta}, S_{0}\right\}:=M_{2}$,

$\frac{d}{d t} S(t) \leq M_{1} M_{2} \lambda+M_{2} \eta$

Similarly, from the rest of the equations of (1), we obtain $O(t) \leq \max \left\{\frac{M_{2}^{2} \zeta \kappa+(B \zeta+\kappa \xi) M_{2}+B \xi}{\xi}, O_{0}\right\}:=M_{3}$,

$\frac{d}{d t} O(t) \leq M_{2} M_{3} \zeta+M_{3} \xi$,

and

$H(t) \leq \frac{M_{2} c}{d}:=M_{4}$,

$\frac{d}{d t} H(t) \leq c M_{2}$.

The proof is complete. 


\section{Equilibrium Points: Existence AND ANALYSIS}

To find equilibrium points of system (1), we set the derivative equal to zero and then solve for $C^{*}, S^{*}, O^{*}$, and $S^{*}$. It is easily to find that the first five equilibrium points of system (1) are as follow:

$E_{1}=(0,0,0,0)$

$E_{2}=\left(C^{*}, 0,0,0\right)$, where $C^{*}=\frac{(\beta-\mu) K}{\beta}, \beta>\mu$,

$E_{3}=\left(0, S^{*}, 0, H^{*}\right)$, where $S^{*}=\frac{\eta Q d}{Q c \sigma+d \eta}, H^{*}=\frac{\eta Q c}{Q c \sigma+d \eta}$,

$E_{4}=\left(0,0, O^{*}, 0\right)$, where $O^{*}=B$,

$E_{5}=\left(C^{*}, 0, O^{*}, 0\right)$, where $C^{*}=\frac{K(\beta-\mu)}{\beta}, O^{*}=B$.

To solve for the rest of equilibrium points, i.e., $E_{6}=\left(C^{*}, S^{*}, 0, H^{*}\right) \quad, \quad E_{7}=\left(0, S^{*}, O^{*}, H^{*}\right) \quad, \quad$ and $E_{8}=\left(C^{*}, S^{*}, O^{*}, H^{*}\right)$ which are characterized by 3 and 4 systems of equation, we begin by writing $C, O$, and $H$ as functions of $S$ and then substitute them into the $S$ equation so that we can solve for $S$.

For $E_{6}$ which has $O=0$, we set the derivatives equal zero in the first, second, and fourth equation of (1) and obtain

$0=\beta C^{*}\left(1-\frac{C^{*}}{K}\right)-\gamma C^{*} S^{*}-\mu C^{*}$,

$0=\eta S^{*}\left(1-\frac{S^{*}}{C^{*} \omega+Q}\right)+\lambda C^{*} S^{*}-\sigma S^{*} H^{*}$

$0=c S^{*}-d H^{*}$.

Therefore, solving for $H^{*}$ and $C^{*}$ as functions of $S^{*}$, we obtain

$C^{*}=\frac{K\left(\beta-S^{*} \gamma-\mu\right)}{\beta}$,

$H^{*}=\frac{c S^{*}}{d}$.

Then substituting these formulas into (4), we find

$A_{1} S^{* 2}+A_{2} S^{*}+A_{3}=0$

where

$$
\begin{aligned}
A_{1}= & K^{2} d \gamma^{2} \lambda \omega+K \beta c \gamma \omega \sigma, \\
A_{2}= & 2 K^{2} d \gamma \lambda \mu \omega+K \beta c \mu \omega \sigma-\left(2 K^{2} \beta d \gamma \lambda \omega+K Q \beta d \gamma \lambda+K \beta^{2} c \omega \sigma\right. \\
& \left.+K \beta d \eta \gamma \omega+Q \beta^{2} c \sigma+\beta^{2} d \eta\right), \\
A_{3}= & K^{2} \beta^{2} d \lambda \omega+K^{2} d \lambda \mu^{2} \omega+K Q \beta^{2} d \lambda+K \beta^{2} d \eta \omega \\
& +Q \beta^{2} d \eta-\left(2 K^{2} \beta d \lambda \mu \omega+K Q \beta d \lambda \mu-K \beta d \eta \mu \omega\right) .
\end{aligned}
$$

The $S$ solution can be found from $S^{*}=\frac{-A_{2} \pm \sqrt{A_{2}^{2}-4 A_{1} A_{3}}}{2 A_{1}}$. Since $A_{1}$ is always positive, thus if $A_{2}, A_{3}>0, S$ does not exist. If $A_{2}<0, A_{3}>0$, two $S^{*}$ exists when $A_{2}^{2}>4 A_{1} A_{3}$. If $A_{2} A_{3}<0$, unique $S^{*}$ exists.

Repeating the same process, we obtain $S$ for $E_{7}$ and $E_{8}$ respectively as below

$A_{1} S^{* 2}+A_{2} S^{*}+A_{3}=0$

where $A_{1}=Q \zeta d \delta \kappa, A_{2}=B Q \zeta d \delta+Q d \delta \kappa \xi+Q c \sigma \xi+d \eta \xi$, and $A_{3}=B Q d \delta \xi-\eta \xi d Q$.

and

$A_{1} S^{* 2}+A_{2} S^{*}+A_{3}=0$

where

$$
\begin{aligned}
A_{1}= & B K \zeta \beta d \delta \gamma \omega+K^{2} d \gamma^{2} \lambda \omega \xi+K \zeta \beta d \delta \kappa \mu \omega+K \beta d \delta \gamma \kappa \omega \xi \\
& +K \beta c \gamma \omega \sigma \xi-\left(K \zeta \beta^{2} d \delta \kappa \omega+Q \zeta \beta^{2} d \delta \kappa\right)
\end{aligned}
$$

$A_{2}=K \beta c \mu \omega \sigma \xi+B K \zeta \beta d \delta \mu \omega+K \beta d \delta \kappa \mu \omega \xi+2 K^{2} \gamma d \lambda \mu \omega \xi+B K \gamma \beta d \delta \omega \xi$

$-\left(B K \zeta \beta^{2} d \delta \omega+K \gamma \beta d \eta \omega \xi+Q \beta^{2} d \delta \kappa \xi+2 K^{2} \gamma \beta d \lambda \omega \xi+K \beta^{2} d \delta \kappa \omega \xi\right.$ $\left.+Q \beta^{2} c \sigma \xi+K \beta^{2} c \omega \sigma \xi+\beta^{2} d \eta \xi+K \gamma Q \beta d \lambda \xi+B Q \zeta \beta^{2} d \delta\right)$,

$A_{3}=B K \beta d \delta \mu \omega \xi+K^{2} \beta^{2} d \lambda \omega \xi+K^{2} d \lambda \mu^{2} \omega \xi+K Q \beta^{2} d \lambda \xi$

$$
\begin{aligned}
& +K \beta^{2} d \eta \omega \xi+Q \beta^{2} d \eta \xi-\left(B K \beta^{2} d \delta \omega \xi+2 K^{2} \beta d \lambda \mu \omega \xi\right. \\
& \left.+B Q \beta^{2} d \delta \xi+K Q \beta d \lambda \mu \xi+K \beta d \eta \mu \omega \xi\right) .
\end{aligned}
$$

Similarly $E_{7}$ and $E_{8}$ will exist if there exist positive solutions of $s^{*}$ in (7) or (8). Therefore we have the following equilibrium points:

$$
\begin{gathered}
E_{6}=\left(C^{*}, S^{*}, 0, H^{*}\right), \text { where } C^{*}=\frac{K\left(\beta-S^{*} \gamma-\mu\right)}{\beta}, \\
H^{*}=\frac{c S^{*}}{d}, \beta>\left(S^{*} \gamma+\mu\right), \\
E_{7}=\left(0, S^{*}, O^{*}, H^{*}\right), \text { where } O^{*}=\frac{\zeta \kappa S^{* 2}+(B \zeta+\kappa \xi) S^{*}+B \xi}{\xi}, \\
H^{*}=\frac{S^{*} c}{d}, \\
E_{8}=\left(C^{*}, S^{*}, O^{*}, H^{*}\right), \text { where } C^{*}=\frac{K\left(\beta-S^{*} \gamma-\mu\right)}{\beta}, \\
O^{*}=\frac{\zeta \kappa S^{* 2}+(B \zeta+\kappa \xi) S^{*}+B \xi}{\xi}, H^{*}=\frac{S^{*} c}{d} .
\end{gathered}
$$

\section{Local Stability ANd Hopf BIFURCATION}

As a first example, we will consider the system without barn owls to make adjustment about the squirrel hunters capacity in controlling squirrels when there do not exist barn owls and also to consider the sensitivity of solutions on changing the value of time delay $\tau$, i.e., we consider the system with only one time delay $\tau$. Thus system (1) becomes 
$\frac{d C(t)}{d t}=\beta C(t)\left(1-\frac{C(t)}{K}\right)-\gamma C(t) S(t)-\mu C(t)$,

$\frac{d S(t)}{d t}=\eta S(t)\left(1-\frac{S(t)}{\omega C(t)+Q}\right)+\lambda C(t-\tau) S(t)-\sigma S(t) H(t)$,

$\frac{d H(t)}{d t}=c S(t)-d H(t)$,

To meet the biological sense, the coexist equilibrium point $E_{80}=\left(C^{*}, S^{*}, H^{*}\right)$ of (9) will be analyzed. Thus the Jacobian matrix of (9) is given by

$\left[\begin{array}{ccc}a_{11} & a_{12} & 0 \\ a_{21} & a_{22} & a_{23} \\ 0 & a_{32} & a_{33}\end{array}\right]$

where

$a_{11}=\beta\left(1-\frac{C^{*}}{K}\right)-\frac{\beta C^{*}}{K}-\lambda S^{*}-\mu$,

$a_{12}=-\lambda C^{*}, a_{21}=\frac{\eta S^{* 2} \omega}{\left(C^{*} \omega+Q\right)^{2}}+e^{-\lambda \tau} \lambda S^{*}$,

$a_{22}=\eta\left(1-\frac{S^{*}}{C^{*} \omega+Q}\right)-\frac{\eta S^{*}}{C^{*} \omega+Q}+\lambda C^{*}-\sigma H^{*}$,

$a_{23}=-\sigma S^{*}, a_{32}=c, a_{33}=-d$.

The characteristic equation of (10) is

$V_{1} \Lambda^{2}+V_{2} \Lambda+V_{3}+V_{4} e^{-\Lambda \tau}=0$,

where

$$
\begin{aligned}
V_{1}= & \sigma H^{*}+\gamma S^{*}+d+\mu\left(2 a_{1} \beta+2 a_{2} \eta-\lambda C^{*}-\beta-\eta\right), \\
V_{2}= & \left(\gamma a_{2}^{2} \eta \omega C^{*}+2 \gamma a_{2} \eta S^{*}-\gamma \lambda C^{*} S^{*}\right)+\left(4 a_{1} a_{2} \beta \eta-2 a_{2} \beta \eta\right) \\
& +\left(d \sigma H^{*}+\mu \sigma H^{*}-\beta \sigma H^{*}\right)+\left(\gamma d S^{*}+c \sigma S^{*}-\gamma \eta S^{*}\right) \\
& +\left(2 a_{1} \beta d-2 a_{1} \beta \eta\right)+\left(\beta \lambda C^{*} 2 a_{2} d \eta-d \eta-d \lambda C^{*}\right)+\left(2 a_{2} \eta \mu-\eta \mu\right) \\
& +(\beta \eta+d \mu-\beta d)+\left(\gamma S^{*} H^{*} \sigma+2 a_{1} \beta \sigma H^{*}-2 a_{1} \beta \lambda C^{*}-\mu \lambda C^{*}\right), \\
V_{3}= & \left(\gamma c \sigma S^{*}-\gamma d \eta S^{*}\right)+\left(c \sigma \mu S^{*}+\beta d \lambda C^{*}-\mu d \lambda C^{*}-c \sigma \beta S^{*}\right) \\
& +\left(2 a_{2} d \eta \mu+d \eta \beta-d \eta \mu-2 a_{2} d \eta \beta\right)+\left(d \sigma \mu H^{*}+4 a_{1} a_{2} \beta d \eta-d \sigma \beta H^{*}\right) \\
& +\left(\gamma a_{2}^{2} d \eta \omega C^{*}+2 a_{1} \beta c \sigma S^{*}-2 a_{1} \beta d \eta-\gamma d \lambda C^{*} S^{*}\right) \\
& +\left(2 a_{I} \beta d \sigma H^{*}+2 \gamma a_{2} d \eta S^{*}+\gamma d \sigma S^{*} H^{*}-2 a_{1} \beta d \lambda C^{*}\right), \\
V_{4} & =\gamma d \lambda C^{*} S^{*}+\gamma \lambda^{2} C^{*} S^{*} .
\end{aligned}
$$

By choosing $\tau$ as the bifurcation parameter, we study the stability of the equilibrium and the Hopf bifurcation of system (9) by separating it into two cases.

For case 1: $\tau=0$. The characteristic equation (11) reduces to a third order polynomial equation,

$$
\Lambda^{3}+V_{1} \Lambda^{2}+V_{2} \Lambda+G_{1}=0,
$$

where $G_{1}=V_{3}+V_{4}$.

According to Routh-Hurwitz criterion, if
$V_{i}>0, \quad i=1,2,3,4$ and $V_{1} V_{2}-G_{1}>0$,

then all roots have negative real parts.

Thus, the equilibrium $E_{80}=\left(C^{*}, S^{*}, H^{*}\right)$ of system (9) is locally asymptotically stable if the conditions in (13) are satisfied.

For case 2: $\tau>0$, the characteristic equation (11) becomes $\Lambda^{3}+V_{1} \Lambda^{2}+V_{2} \Lambda+V_{3}+V_{4} e^{-\Lambda \tau}=0$.

We now look for a possible Hopf bifurcation which could occur if a purely imaginary complex conjugate pair of eigenvalues exists. Defining $\lambda= \pm i \omega$, where $\omega$ is real and positive, and separating real and imaginary parts of the characteristic equation (14), we have

$-V_{1} \omega^{2}+V_{4} \cos (\omega \tau)+V_{3}=0$,

$-\omega^{3}-\sin (\omega \tau) V_{4}+V_{2} \omega=0$.

Squaring both sides of the real and imaginary parts in (15) and adding, we obtain a formula for $\omega$ as follows.

$\omega^{6}+G_{2} \omega^{4}+G_{3} \omega^{2}+G_{4}=0$,

where $G_{2}=V_{1}^{2}-2 V_{2}, G_{3}=V_{2}^{2}-2 V_{1} V_{3}, G_{4}=V_{3}^{2}-V_{4}^{2}$.

A Hopf bifurcation can only exist if $\omega^{2}$ is real and positive. By Descartes Rule of Signs, if one of the following sets of conditions [17]:

$G_{2}>0, G_{4}<0$,

$G_{3}<0, G_{4}<0$,

hold. Then (16) has at least one positive real root $\omega^{* 2}$.

Substituting $\omega^{*}$ into (15), we obtain

$\tan \left(\omega^{*} \tau^{c}\right)=\frac{-\omega^{* 3}+V_{2} \omega}{V_{1} \omega^{* 2}-V_{3}}$,

which leads to

$\tau_{h}^{c}=\frac{1}{\omega^{*}} \arctan \frac{-\omega^{* 3}+V_{2} \omega^{*}}{V_{1} \omega^{* 2}-V_{3}}+\frac{1}{\omega^{*}} 2 h \pi, \quad h=0,1,2$.

Next, we will prove $\Lambda(\tau)$ meets the transversality conditions; taking the derivative of system (14) with respect to $\tau$, one derives that

$\left[\frac{d \Lambda}{d \tau}\right]^{-1}=\frac{-2 V_{1} \Lambda-3 \Lambda^{2}-V_{2}}{\Lambda\left(V_{I} \Lambda^{2}+\Lambda^{3}+V_{2} \Lambda+V_{3}\right)}-\frac{\tau}{\Lambda}$.

So, if

$\operatorname{Re}\left[\frac{d \Lambda}{d \tau}\right]_{\Lambda=i \omega}^{-1}=\frac{3 \omega^{5}+A_{1} \omega^{3}+A_{2} \omega}{\omega^{7}+A_{3} \omega^{5}+A_{4} \omega^{3}-A_{5} \omega}>0$,

where $A_{1}=-2 V_{1}^{2}-4 V_{2}, A_{2}=2 V_{1} V_{3}+V_{2}^{2}, A_{3}=-V_{1}^{2}-2 V_{2}$, $A_{4}=2 V_{1} V_{3}+V_{2}^{2}, A_{5}=V_{3}^{2}$,

holds, then the root $\Lambda$ of (14) crosses the imaginary axis at $\tau=\tau_{h}^{c}$ from left to right. 
These two conditions prove that a Hopf bifurcation could exist at $\tau_{h}^{c}$. However, to prove that the point is actually a Hopf bifurcation it would also be necessary to prove that the $E_{80}=\left(C^{*}, S^{*}, H^{*}\right)$ equilibrium is stable for all $\tau<\tau_{h}^{c}$.

We now consider the system with barn owl (1) with the non-zero equilibrium $E_{8}$. By linearization, the corresponding characteristic equation of system (1) becomes

$$
\begin{aligned}
& \Lambda^{4}+A_{1} \Lambda^{3}+A_{2} \Lambda^{2}+A_{3} \Lambda+A_{4}+\left(A_{5} \Lambda^{2}+A_{6} \Lambda-A_{7}\right) e^{-\lambda \tau_{1}} \\
& +\left(A_{8} \Lambda^{2}+A_{9} \Lambda+A_{10}\right) e^{-\lambda \tau_{2}}=0,
\end{aligned}
$$

where

$$
\begin{aligned}
A_{1}= & 2 a_{3} \xi+b_{1}+b_{2}+d-\left(b_{3}+b_{4}+\beta\right), \\
A_{2}= & a_{2}^{2} \eta \gamma \omega C^{*}+a_{3}^{2} \delta \kappa \xi S^{*}+c \sigma S^{*}+2 a_{3} b_{1} \xi+2 a_{3} b_{2} \xi \\
& +2 a_{3} d \xi+b_{1} b_{2}+b_{2} d_{4}+b_{3} \beta+b_{1} d+b_{4} \beta \\
& -\left(2 a_{3} b_{3} \xi+2 a_{3} \beta \xi+b_{1} b_{3}+b_{1} b_{4}+b_{2} b_{4}+b_{2} \beta+b_{3} d+b_{4} d+\beta d\right),
\end{aligned}
$$$$
A_{3}=2 \gamma a_{2}^{2} a_{3} \eta \omega \xi C^{*}+d b_{2} b_{1}+2 a_{3} b_{1} b_{2} \xi+2 a_{3} b_{3} \beta \xi+2 d a_{3} b_{1} \xi
$$$$
+2 d a_{3} b_{2} \xi+b_{l} c \sigma S^{*}+d b_{3} b_{4}+d b_{3} \beta+d b_{4} \beta+b_{1} b_{3} b_{4}+b_{2} b_{4} \beta
$$$$
+2 S^{*}{ }^{*} c \sigma \xi+d a_{3}^{2} \delta \kappa \xi S^{*}+d \gamma a_{2}^{2} \eta \omega C^{*}+a_{3}^{2} b_{1} \delta \kappa \xi S^{*}\left(b_{1} b_{2} b_{4}\right.
$$$$
+d b_{3} b_{1}+d b_{1} b_{4}+d b_{2} b_{4}+d b_{2} \beta+b_{3} b_{4} \beta+\gamma a_{2}^{2} b_{4} \eta \omega C^{*}+a_{3}^{2} \beta \delta \kappa \xi S^{*}
$$$$
\left.+2 a_{3} b_{1} b_{3} \xi+2 a_{3} b_{2} \beta \xi+2 d a_{3} b_{3} \xi+2 d a_{3} \beta \xi+b_{4} c \sigma S^{*}+\beta c \sigma S^{*}\right),
$$$$
A_{4}=+c \sigma b_{4} \beta S^{*}+2 d a_{3} b_{3} \beta \xi+d a_{3}^{2} b_{1} \delta \kappa \xi S^{*}+d b_{1} b_{3} b_{4}+d b_{2} b_{4} \beta
$$$$
+2 d \gamma a_{2}^{2} a_{3} \eta \omega \xi C^{*}+2 d a_{3} b_{1} b_{2} \xi+2 c \sigma a_{3} b_{1} \xi S^{*}-\left(2 d a_{3} b_{1} b_{3} \xi\right.
$$$$
+2 c \sigma a_{3} \beta \xi S^{*}+d a_{3}^{2} \beta \delta \kappa \xi S^{*}+2 d a_{3} b_{2} \beta \xi+d \gamma a_{2}^{2} b_{4} \eta \omega C^{*}
$$$$
\left.+d b_{1} b_{2} b_{4}+c \sigma b_{1} b_{4} S^{*}+d b_{3} b_{4} \beta\right),
$$

$A_{5}=\gamma \lambda C^{*} S^{*} \Lambda^{2}, \quad A_{6}=2 a_{3} \gamma \lambda \xi C^{*} S^{*}+d \gamma \lambda C^{*} s^{*}-b_{4} \gamma \lambda C^{*} s^{*}$, $A_{7}=2 d \gamma a_{3} \lambda \xi C^{*} S^{*}-d \gamma b_{4} \lambda C^{*} S^{*}, A_{8}=\delta \zeta O^{*} s^{*}$,

$A 9=b_{1} \delta \zeta O^{*} S^{*}+d \delta \zeta O^{*} S^{*}-\beta \delta \zeta O^{*} S^{*}$,

$A_{10}=d b_{1} \delta \zeta O^{*} S^{*}-d \beta \delta \zeta O^{*} S^{*}, \quad b_{1}=\gamma S^{*}+a_{1} \beta+\mu$, $b_{2}=\sigma H^{*}+\delta O^{*}+n a_{2}, \quad b_{3}=\lambda C^{*}+\eta, \quad b_{4}=\zeta S^{*}+\xi$.

Next, we consider the local stability of positive equilibrium $E_{8}=\left(C^{*}, S^{*}, O^{*}, H^{*}\right)$ and the Hopf bifurcation of system (1) for different combinations of $\tau_{1}$ and $\tau_{2}$.

Case 1: $\tau_{1}=\tau_{2}=0$. (17) becomes

$$
\lambda^{4}+B_{1} \lambda^{3}+B_{2} \lambda^{2}+B_{3} \lambda+B_{4}=0,
$$

where $\quad B_{1}=A_{1} \quad, \quad B_{2}=A_{2}+A_{5}+A_{8} \quad, \quad B_{3}=A_{3}+A_{6}+A_{9}$, $B_{4}=A_{10}+A_{4}+A_{7}$.
By Routh-Hurwitz criteria, $E_{8}=\left(C^{*}, S^{*}, 0, H^{*}\right)$ is locally asymptotically stable if the following conditions $B_{1}>0, B_{1} B_{2}-B_{3}>0, B_{3}\left(B_{1} B_{2}-B_{3}\right)-B_{1}^{2} B_{4}>0, B_{4}>0$ hold.

Case 2: $\tau_{1}>0, \tau_{2}=0$. On substituting $\tau_{2}=0,(17)$ becomes $\Lambda^{4}+A_{1} \Lambda^{3}+A_{2} \Lambda^{2}+A_{3} \Lambda+A_{4}+V_{1} e^{-\Lambda \tau_{1}}+A_{8} \Lambda^{2}+A_{9} \Lambda+A_{10}=0$. (19)

Then setting $\lambda= \pm i \omega_{1}$ and separating real and imaginary parts, we obtain

$$
\begin{aligned}
& A_{6} \sin \left(\omega_{1} \tau_{1}\right) \omega_{1}-A_{5} \cos \left(\omega_{1} \tau_{1}\right) \omega_{1}^{2}+\omega_{1}^{4}-A_{2} \omega_{1}^{2}-A_{8} \omega_{1}^{2} \\
& +A_{7} \cos \left(\omega_{1} \tau_{1}\right)+A_{10}+A_{4}=0, \\
& -A_{1} \omega_{1}^{3}+A_{5} \sin \left(\omega_{1} \tau_{1}\right) \omega_{1}^{2}+A_{6} \cos \left(\omega_{1} \tau_{1}\right) \omega_{1}+A_{3} \omega_{1} \\
& -A_{7} \sin \left(\omega_{1} \tau_{1}\right)+A_{9} \omega_{1}=0 .
\end{aligned}
$$

Squaring both sides of the real and imaginary parts and adding, we have the equation for $\omega_{1}$ as

$\omega_{1}^{8}+B_{7} \omega_{1}^{6}+B_{8} \omega_{1}^{4}+B_{9} \omega_{1}^{2}+B_{10}=0$

where

$B_{7}=A_{1}^{2}-2\left(A_{2}+A_{8}\right)$,

$B_{8}=A_{2}^{2}+A_{8}^{2}+2\left(A_{10}+A_{4}+A_{2} A_{8}\right)-2\left(A_{1} A_{3}+A_{1} A_{9}\right)-A_{5}^{2}$,

$B_{9}=A_{3}^{2}+A_{9}^{2}+2\left(A_{3} A_{9}+A_{5} A_{7}\right)-2\left(A_{10} A_{2}+A_{10} A_{8}+A_{2} A_{4}+A_{4} A_{8}\right)-A_{6}^{2}$,

$B_{10}=A_{10}^{2}+2 A_{10} A_{4}+A_{4}^{2}-A_{7}^{2}$.

If one set of the following sets of conditions

$B_{7}>0, B_{8}>0, B_{10}<0$,

$B_{7}>0, B_{9}<0, B_{10}<0$,

$B_{8}<0, B_{9}<0, B_{10}<0$,

holds, then (21) has at least one positive root $\omega_{1}^{*}$.

Substituting $\omega_{1}^{*}$ into (20), we obtain

$$
\tau_{1 h}^{c}=\frac{1}{\omega_{1}^{*}} \arccos \frac{D_{1} \omega_{1}^{* 6}+D_{2} \omega_{1}^{* 4}+D_{3} \omega_{1}^{* 2}+D_{4}}{D_{5} \omega_{1}^{* 4}+D_{6} \omega_{1}^{* 2}+D_{7}}+\frac{2 \pi h}{\omega_{1}^{*}}
$$

where $h=0,1,2, \ldots$ and

$D_{1}=A_{5}, \quad D_{2}=A_{1} A_{6}-A_{2} A_{5}-A_{5} A_{8}-A_{7}$,

$D_{3}=A_{10} A_{5}+A_{2} A_{7}+A_{4} A_{5}+A_{7} A_{8}-A_{3} A_{6}-A_{6} A_{9}$,

$D_{4}=-A_{10} A_{7}-A_{4} A_{7}, \quad D_{5}=A_{5}^{2}, \quad D_{6}=A_{6}^{2}-2 A_{5} A_{7}, D_{7}=A_{7}^{2}$.

Differentiating $\Lambda$ w.r.t. $\tau_{1}$ and using (19), we obtain $\left[\frac{d \Lambda}{d \tau_{1}}\right]^{-1}=\frac{2 A_{5} \Lambda+A_{6}}{\left(A_{5} \Lambda^{2}+A_{6} \Lambda+A_{7}\right) \Lambda}$ $-\frac{3 A_{1} \Lambda^{2}+4 \Lambda^{3}+2 A_{2} \Lambda+2 A_{8} \Lambda+A_{3}+A_{9}}{\Lambda\left(A_{1} \Lambda^{3}+\Lambda^{4}+A_{2} \Lambda^{2}+A_{8} \Lambda^{2}+A_{3} \Lambda+A_{9} \Lambda+A_{10}+A_{4}\right)}-\frac{\tau_{1}}{\Lambda}$,

which lead to 
$\operatorname{Re}\left[\frac{d \Lambda}{d \tau_{1}}\right]_{\Lambda=i o_{1}^{*}}^{-1}=\frac{P_{2 I} Q_{2 I}+P_{2 R} Q_{2 R}}{Q_{2 I}^{2}+Q_{2 R}^{2}}$,

where

$$
\begin{aligned}
P_{2 I}= & \left(A_{1} A_{5}+3 A_{6}\right) \omega^{4}+\left(A_{3} A_{5}+A_{5} A_{9}-\left(3 A_{1} A_{7}+A_{2} A_{6}\right.\right. \\
& \left.\left.+A_{6} A_{8}\right)\right) \omega^{2}+A_{3} A_{7}+A_{7} A_{9}-\left(A_{10} A_{6}+A_{4} A_{6}\right), \\
P_{2 R}= & -2 A_{5} \omega^{5}+\left(2 A_{1} A_{6}+4 A_{7}\right) \omega^{3}+\left(2 A_{10} A_{5}+2 A_{4} A_{5}\right. \\
& \left.-\left(2 A_{2} A_{7}+2 A_{7} A_{8}\right)\right) \omega,
\end{aligned}
$$

$Q_{2 I}=\left(A_{1} A_{5}+A_{6}\right) \omega^{6}+\left(-A_{1} A_{7}-A_{2} A_{6}-A_{3} A_{9}-A_{5} A_{9}-A_{6} A_{8}\right) \omega^{4}$

$$
+\left(A_{10} A_{6}+A_{3} A_{7}+A_{4} A_{6}+A_{7} A_{9}\right) \omega^{2},
$$$$
+\left(-A_{10} A_{5}-A_{2} A_{7}-A_{3} A_{6}-A_{4} A_{9}-A_{1} A_{8}\right) \omega^{3}
$$$$
+\left(A_{10} A_{7}+A_{4} A_{7}\right) \omega
$$

\section{If}

$\mathrm{P}_{2 \mathrm{I}} \mathrm{Q}_{2 \mathrm{I}}+\mathrm{P}_{2 \mathrm{R}} \mathrm{Q}_{2 \mathrm{R}}>0$,

holds, then the root $\Lambda$ of (19) crosses the imaginary axis at $\tau_{1}=\tau_{1}^{c}$ from left to right.

Case 3: $\tau_{1}=0, \tau_{2}>0$. On substituting $\tau_{1}=0$, (17) becomes $\Lambda^{4}+A_{1} \Lambda^{3}+A_{2} \Lambda^{2}+A_{3} \Lambda+A_{4}+A_{5} \Lambda^{2}+A_{6} \Lambda+A_{7}+V_{2} e^{-\Lambda \tau_{2}}=0$.

Defining $\Lambda=i \omega_{2}$ and separating real and imaginary parts, we have

$$
\begin{aligned}
& A_{9} \sin \left(\omega_{2} \tau_{2}\right) \omega_{2}-A_{8} \cos \left(\omega_{2} \tau_{2}\right) \omega_{2}^{2}+\omega_{2}^{4}-A_{2} \omega_{2}^{2} \\
& -A_{5} \omega_{2}^{2}+A_{10} \cos \left(\omega_{2} \tau_{2}\right)+A_{4}+A_{7}=0, \\
& -A_{1} \omega_{2}^{3}+A_{8} \sin \left(\omega_{2} \tau_{2}\right) \omega_{2}^{2}+A_{9} \cos \left(\omega_{2} \tau_{2}\right) \omega_{2} \\
& -A_{10} \sin \left(\omega_{2} \tau_{2}\right)+A_{3} \omega_{2}+A_{6} \omega=0 .
\end{aligned}
$$

Squaring both sides of the real and imaginary parts and adding, we obtain

$$
\omega_{2}^{8}+B_{11} \omega_{2}^{6}+B_{12} \omega_{2}^{4}+B_{13} \omega_{2}^{2}+B_{14}=0,
$$

where

$$
\begin{aligned}
B_{11}= & A_{1}^{2}-\left(2 A_{2}+2 A_{5}\right), B_{12}=A_{2}^{2}+2 A_{2} A_{5}+A_{5}^{2} \\
& +2 A_{4}+2 A_{7}-\left(2 A_{1} A_{3}+2 A_{1} A_{6}+A_{8}^{2}\right), \\
B_{13}= & 2 A_{10} A_{8}+A_{3}^{2}+2 A_{3} A_{6}+A_{6}^{2}-\left(2 A_{2} A_{4}+2 A_{2} A_{7}\right. \\
& \left.+2 A_{4} A_{5}+2 A_{5} A_{7}+A_{9}^{2}\right), \\
B_{14}= & A_{4}^{2}+2 A_{4} A_{7}+A_{7}^{2}-A_{10}^{2} .
\end{aligned}
$$

If one set of the following sets of conditions $B_{11}>0, B_{12}>0, B_{14}<0$, $B_{11}>0, B_{13}<0, B_{14}<0$, $B_{12}<0, B_{13}<0, B_{14}<0$, hold, then (24) has at least one positive root $\omega_{2}^{*}$. Substituting $\omega_{2}^{*}$ into (23), we obtain$$
Q_{2 R}=-A_{5} \omega^{7}+\left(A_{1} A_{6}+A_{2} A_{5}+A_{5} A_{8}+A_{7}\right) \omega^{5}
$$

$\tau_{2 h}^{c}=\frac{1}{\omega_{2}^{*}} \arccos \frac{D_{8} \omega_{2}^{* 6}+D_{9} \omega_{2}^{* 4}+D_{10} \omega_{2}^{* 2}+D_{11}}{D_{12} \omega_{2}^{* 4}+D_{13} \omega_{2}^{* 2}+D_{14}}+\frac{2 \pi h}{\omega_{2}^{*}}$,

where

$D_{8}=A_{8}, D_{9}=A_{1} A_{g}-A_{2} A_{8}-A_{5} A_{8}-A_{10}$,

$D_{10}=A_{10} A_{2}+A_{10} A_{5}+A_{4} A_{8}+A_{7} A_{8}-A_{3} A_{9}-A_{6} A_{9}$,

$D_{11}=-A_{10} A_{4}-A_{10} A_{7}, D_{12}=A_{8}^{2}$,

$D_{13}=A_{9}^{2}-2 A_{10} A_{8}, D_{14}=A_{10}^{2}$.

Differentiating $\Lambda$ w.r.t. $\tau_{2}$ and using (22), we obtain

$$
\begin{aligned}
& {\left[\frac{d \Lambda}{d \tau_{2}}\right]^{-1}=\frac{2 A_{8} \Lambda+A_{9}}{\left(A_{8} \Lambda^{2}+A_{9} \Lambda+A_{10}\right) \Lambda}} \\
& -\frac{3 A_{1} \Lambda^{2}+4 \Lambda^{3}+2 A_{2} \Lambda+2 A_{5} \Lambda+A_{3}+A_{6}}{\Lambda\left(A_{1} \Lambda^{3}+\Lambda^{4}+A_{2} \Lambda^{2}+A_{5} \Lambda^{2}+A_{3} \Lambda+A_{6} \Lambda+A_{4}+A_{7}\right)}-\frac{\tau_{2}}{\Lambda},
\end{aligned}
$$

which leads to

$$
\operatorname{Re}\left[\frac{d \Lambda}{d \tau_{2}}\right]_{\Lambda=i \omega_{2}^{*}}^{-1}=\frac{P_{3 I} Q_{31}+P_{3 R} Q_{3 R}}{Q_{3 I}^{2}+Q_{3 R}^{2}}
$$

where

$$
\begin{aligned}
P_{3 I}= & \left(-5 A_{l} A_{8}-5 A_{9}\right) \omega^{4}+\left(3 A_{1} A_{10}+3 A_{2} A_{9}+3 A_{3} A_{8}+3 A_{5} A_{9}\right. \\
& \left.+3 A_{6} A_{8}\right) \omega^{2}-A_{10} A_{3}-A_{10} A_{6}-A_{4} A_{9}-A_{7} A_{9}, \\
P_{3 R}= & 6 A_{8} \omega^{5}+\left(-4 A_{1} A_{9}-4 A_{2} A_{8}-4 A_{10}\right) \omega^{3}+\left(2 A_{10} A_{2}+2 A_{10} A_{5}\right. \\
& \left.+2 A_{3} A_{9}+2 A_{4} A_{8}+2 A_{6} A_{9}+2 A_{7} A_{8}\right) \omega, \\
Q_{3 I}= & \left(-A_{1} A_{8}-A_{9}\right) \omega^{6}+\left(A_{1} A_{10}+A_{2} A_{8}+A_{3} A_{9}+A_{6} A_{8}\right) \omega^{4} \\
& +\left(-A_{10} A_{3}-A_{10} A_{6}-A_{4} A_{9}-A_{7} A_{9}\right) \omega^{2}, \\
Q_{3 R}= & A_{8} \omega^{7}+\left(-A_{1} A_{9}-A_{2} A_{8}-A_{5} A_{8}-A_{10}\right) \omega^{5} \\
& +\left(A_{10} A_{2}+A_{10} A_{5}+A_{3} A_{8}+A_{6} A_{8}+A_{1} A_{8}\right) \omega^{3} \\
& +\left(-A_{10} A_{4}-A_{10} A_{7}\right) \omega .
\end{aligned}
$$

If the following condition

$P_{3 I} Q_{3 I}+P_{3 R} Q_{3 R}>0$,

holds, then the root $\Lambda$ of (22) crosses the imaginary axis at $\tau_{2}=\tau_{2}^{c}$ from left to right.

Case 4: $\tau_{1}=\tau_{2}=\tau>0$. By substituting $\tau_{1}=\tau_{2}=\tau$, (17) becomes $\Lambda^{4}+A_{1} \Lambda^{3}+A_{2} \Lambda^{2}+A_{3} \Lambda+A_{4}+V_{1} e^{-\Lambda \tau}+V_{2} e^{-\Lambda \tau}=0$.

Defining $\Lambda= \pm i \omega_{3}$ and separating real and imaginary parts, we obtain

$$
\begin{aligned}
& A_{6} \sin \left(\omega_{3} \tau\right) \omega_{3}+A_{0} \sin \left(\omega_{3} \tau\right) \omega_{3}-A_{5} \cos \left(\omega_{3} \tau\right) \omega_{3}^{2} \\
& -A_{8} \cos \left(\omega_{3} \tau\right) \omega_{3}^{2}+\omega_{3}^{4}-A_{2} \omega_{2}^{2}+A_{10} \cos \left(\omega_{3} \tau\right) \\
& +A_{1} \cos \left(\omega_{3} \tau\right)+A_{4}=0,
\end{aligned}
$$




$$
\begin{aligned}
& -A_{1} \omega_{2}^{3}+A_{5} \sin \left(\omega_{3} \tau\right) \omega_{3}^{2}+A_{8} \sin \left(\omega_{3} \tau\right) \omega_{3}^{2} \\
& +A_{6} \cos \left(\omega_{3} \tau\right) \omega_{3}+A_{9} \cos \left(\omega_{3} \tau\right) \omega_{3}-A_{10} \sin \left(\omega_{3} \tau\right) \\
& +A_{3} \omega_{3}-A_{1} \sin \left(\omega_{3} \tau\right)=0 .
\end{aligned}
$$

Squaring both sides and adding, we get

$\omega_{3}^{8}+B_{13} \omega_{3}^{6}+B_{14} \omega_{3}^{4}+B_{15} \omega_{3}^{2}+B_{16}=0$,

where

$B_{13}=A_{1}^{2}+2 A_{2}$,

$B_{14}=A_{2}^{2}-\left(2 A_{1} A_{3}+A_{5}^{2}+2 A_{5} A_{8}+A_{8}^{2}+2 A_{4}\right)$,

$B_{15}=2 A_{10} A_{5}+2 A_{10} A_{8}+A_{3}^{2}+2 A_{5} A_{7}+2 A_{7} A_{8}$

$$
-\left(2 A_{2} A_{4}+A_{6}^{2}+2 A_{6} A_{9}+A_{9}^{2}\right) \text {, }
$$

$B_{16}=-A_{10}^{2}+A_{4}^{2}-\left(2 A_{10} A_{7}+A_{7}^{2}\right)$.

If one set of the following sets of conditions

$B_{15}>0, B_{16}>0, B_{18}<0$,

$B_{15}>0, B_{17}<0, B_{18}<0$,

$B_{16}<0, B_{17}<0, B_{18}<0$,

hold, then (28) has at least one positive root $\omega_{3}^{*}$.

Substituting $\omega_{3}^{*}$ into (27), we obtain

$\tau_{3 h}^{c}=\frac{1}{\omega_{3}^{*}} \arccos \frac{D_{15} \omega_{3}^{* 6}+D_{16} \omega_{3}^{* 4}+D_{17} \omega_{3}^{* 2}+D_{18}}{D_{3} \omega_{3}^{* 4}+D_{20} \omega_{3}^{* 2}+D_{21}}+\frac{2 \pi h}{\omega_{3}^{*}}$,

where

$D_{15}=A_{5}+A_{8}, D_{16}=A_{1} A_{6}+A_{1} A_{9}-\left(A_{2} A_{5}+A_{2} A_{8}+A_{10}+A_{7}\right)$,

$D_{17}=A_{10} A_{2}+A_{2} A_{7}+A_{4} A_{5}+A_{4} A_{\beta}-\left(A_{3} A_{6}+A_{3} A_{9}\right)$,

$D_{18}=-A_{10} A_{4}-A_{4} A_{7} D_{19}=A_{5}^{2}+2 A_{5} A_{8}+A_{8}^{2}$,

$D_{20}=A_{6}^{2}+2 A_{6} A_{9}+A_{9}^{2}-\left(2 A_{10} A_{5}+2 A_{10} A_{8}+2 A_{5} A_{7}+2 A_{7} A_{8}\right)$,

$D_{21}=A_{10}^{2}+2 A_{10} A_{7}+A_{7}^{2}$.

Differentiating $\Lambda$ w.r.t. $\tau_{1}$ and using (26), we obtain

$\left[\frac{d \Lambda}{d \tau}\right]^{-1}=\frac{2 A_{5} \Lambda+2 A_{8} \Lambda+A_{6}+A_{9}}{\Lambda\left(A_{5} \Lambda^{2}+A_{8} \Lambda^{2}+A_{6} \Lambda+A_{9} \Lambda+A_{10}+A_{7}\right)}$

$-\frac{3 A_{1} \Lambda^{2}+4 \Lambda^{3}+2 A_{2} \Lambda+A_{3}}{\Lambda\left(A_{1} \Lambda^{3}+\Lambda^{4}+A_{2} \Lambda^{2}+A_{3} \Lambda+A_{4}\right)}-\frac{\tau}{\Lambda}$,

which leads to

$\operatorname{Re}\left[\frac{d \Lambda}{d \tau}\right]_{\Lambda=i o_{3}^{*}}^{-1}=\frac{P_{4 I} Q_{4 I}+P_{4 R} Q_{4 R}}{Q_{4 I}^{2}+Q_{4 R}^{2}}$,

where

$$
\begin{aligned}
P_{4 I}= & \left(2 A_{5}+2 A_{8}\right) \omega^{5}+\left(-2 A_{1} A_{6}-2 A_{1} A_{9}-4 A_{10}-4 A_{7}\right) \omega^{3} \\
& +\left(2 A_{10} A_{2}+2 A_{2} A_{7}-2 A_{4} A_{5}-2 A_{4} A_{8}\right) \omega, \\
P_{4 R}= & \left(A_{1} A_{5}+A_{1} A_{8}+3 A_{6}+3 A_{9}\right) \omega^{4}+\left(-3 A_{1} A_{10}-3 A_{1} A_{7}-A_{2} A_{6}\right. \\
& \left.-A_{2} A_{9}+A_{3} A_{5}+A_{3} A_{8}\right) \omega^{2}+A_{10} A_{3}+A_{3} A_{7}-A_{4} A_{6}-A_{4} A_{9}, \\
Q_{4 I}= & \left(-A_{5}-A_{8}\right) \omega^{7}+\left(A_{1} A_{6}+A_{1} A_{9}+A_{2} A_{5}+A_{2} A_{8}+A_{10}+A_{7}\right) \omega^{5} \\
& +\left(-A_{10} A_{2}-A_{2} A_{7}-A_{3} A_{6}-A_{3} A_{9}-A_{4} A_{5}-A_{4} A_{8}\right) \omega^{3} \\
& +\left(A_{10} A_{4}+A_{4} A_{7}\right) \omega, \\
Q_{4 R} & =\left(A_{1} A_{5}-A_{1} A_{8}-A_{6}-A_{9}\right) \omega^{6}+\left(A_{1} A_{10}+A_{1} A_{7}+A_{2} A_{6}\right. \\
& \left.+A_{2} A_{9}+A_{3} A_{5}+A_{3} A_{8}\right) \omega^{4} \\
& +\left(-A_{10} A_{3}-A_{3} A_{7}-A_{4} A_{6}-A_{4} A_{9}\right) \omega^{2} .
\end{aligned}
$$

If the following condition

$P_{4 I} Q_{4 I}+P_{4 R} Q_{4 R}>0$,

holds, then the root $\Lambda$ of (26) crosses the imaginary axis at $\tau=\tau_{3}^{c}$ from left to right.

Case 5: $\tau_{1} \neq \tau_{2}, \quad \tau_{1}, \tau_{2}>0$. We consider system (1) with $\tau_{2}$ in its stable interval and regarding $\tau_{1}$ as a parameter.

On substituting $\tau_{1}, \tau_{2}$ into (17), we obtain

$\Lambda^{4}+A_{1} \Lambda^{3}+A_{2} \Lambda^{2}+A_{3} \Lambda+A_{4}+V_{1} e^{-\Lambda \tau_{1}}+V_{2} e^{-\Lambda \tau_{2}}=0$.

Defining $\Lambda= \pm i \omega_{4}$ and separating real and imaginary parts, we have

$A_{6} \sin \left(\omega_{4} \tau_{1}\right) \omega_{4}+A_{0} \sin \left(\omega_{4} \tau_{2}\right) \omega_{4}-A_{s} \cos \left(\omega_{4} \tau_{1}\right) \omega_{4}^{2}$

$-A_{8} \cos \left(\omega_{4} \tau_{2}\right) \omega_{4}^{2}+\omega_{4}^{4}-A_{2} \omega_{4}^{2}+A_{10} \cos \left(\omega_{4} \tau_{2}\right)$

$+A_{1} \cos \left(\omega_{4} \tau_{1}\right)+A_{4}=0$,

$-A_{1} \omega_{4}^{3}+A_{s} \sin \left(\omega_{4} \tau_{1}\right) \omega_{4}^{2}+A_{8} \sin \left(\omega_{4} \tau_{2}\right) \omega_{4}^{2}$

$+A_{6} \cos \left(\omega_{4} \tau_{1}\right) \omega+A_{9} \cos \left(\omega_{4} \tau_{2}\right) \omega-A_{10} \sin \left(\omega_{4} \tau_{2}\right)$

$+A_{3} \omega_{4}-A_{1} \sin \left(\omega_{4} \tau_{1}\right)=0$.

Squaring both sides and adding, we obtain

$\omega_{4}^{8}+B_{17} \omega_{4}^{6}+B_{18} \omega_{4}^{4}+B_{19} \omega_{4}^{2}+B_{20}$

$+\left(-2 A_{8} \omega_{4}^{6}+\left(2 A_{2} A_{8}+2 A_{10}-2 A_{1} A_{9}\right) \omega_{4}^{4}\right.$

$\left.+\left(2 A_{3} A_{9}-2 A_{10} A_{2}-2 A_{4} A_{8}\right) \omega^{2}+2 A_{10} A_{4}\right) \cos \left(\omega_{4} \tau_{2}\right)$

$+\left(\left(2 A_{9}-2 A_{1} A_{8}\right) \omega_{4}^{5}+\left(2 A_{1} A_{10}+2 A_{3} A_{8}-2 A_{2} A_{9}\right) \omega_{4}^{3}\right.$

$\left.+\left(2 A_{4} A_{9}-2 A_{10} A_{3}\right) \omega_{4}\right) \sin \left(\omega_{4} \tau_{2}\right)=0$,

where $B_{19}=A_{1}^{2}-2 A_{2} \quad, \quad B_{20}=A_{2}^{2}-A_{5}^{2}+A_{\delta}^{2}+2 A_{4}-2 A_{1} A_{3} \quad$ ， $B_{21}=A_{3}^{2}+2 A_{5} A_{7}+A_{9}^{2}-A_{6}^{2}-2 A_{10} A_{8}-2 A_{2} A_{4}$ $B_{22}=A_{10}^{2}+A_{4}^{2}-A_{7}^{2}$.

If one set of the following sets of conditions hold: 
$B_{19}>0, B_{20}>0, B_{22}<0$,

$B_{19}>0, B_{21}<0, B_{22}<0$,

$B_{20}<0, B_{21}<0, B_{22}<0$,

then (32) has at least one positive root $\omega_{4}^{*}$. Substituting $\omega_{4}^{*}$ into (31), we obtain

$\tau_{4 h}^{c}=\frac{1}{\omega_{4}^{*}} \arccos \frac{G}{D_{31} \omega_{4}^{* 4}+D_{32} \omega_{4}^{* 2}+D_{33}}+\frac{2 \pi h}{\omega_{4}^{*}}$,

where

$G=D_{22} \omega_{4}^{* 6}+D_{23} \omega_{4}^{* 4}+D_{24} \omega_{4}^{* 2}-D_{25}$

$+\left(D_{26} \omega_{4}^{* 2}-D_{27} \omega_{4}^{* 4}-D_{28}\right) \cos \left(\omega_{4}^{*} \tau_{2}\right)$

$+\left(D_{29} \omega_{4}^{* 3}+D_{30} \omega_{4}^{*}\right) \sin \left(\omega_{4}^{*} \tau_{2}\right), D_{22}=A_{5}$,

$D_{23}=\left(A_{1} A_{6}-A_{2} A_{5}-A_{7}\right), D_{24}=A_{2} A_{7}-A_{3} A_{6}+A_{4} A_{5}$,

$D_{25}=A_{4} A_{7}, D_{26}=A_{10} A_{5}+A_{7} A_{8}-A_{6} A_{9}, \quad D_{27}=A_{5} A_{8}$,

$D_{28}=A_{10} A_{7}, D_{29}=A_{5} A_{\theta}-A_{6} A_{8}$,

$D_{30}=A_{10} A_{6}-A_{7} A_{\theta}, \quad D_{31}=A_{5}^{2}$,

$D_{32}=A_{6}^{2}-2 A_{5} A_{7}, \quad D_{33}=A_{7}^{2}$.

Differentiating $\Lambda$ w.r.t. $\tau_{1}$ and using (30), we obtain

$\left[\frac{d \Lambda}{d \tau_{1}}\right]^{-1}=\frac{2 A_{5} \Lambda+A_{6}}{\Lambda\left(A_{5} \Lambda^{2}+A_{6} \Lambda+A_{7}\right)}-\frac{G_{1} e^{-\Lambda \tau_{2}}+G_{2}}{\left(G_{3}\right) \Lambda e^{-\Lambda \tau_{2}}+\left(G_{4}\right) \Lambda}$,

where $\quad G_{1}=-A_{8} \Lambda^{2} \tau 2-A_{9} \Lambda \tau 2-A_{10} \tau 2+2 A_{8} \Lambda+A_{9}$

$G_{2}=3 A_{1} \Lambda^{2}+4 \Lambda^{3}+2 A_{2} \Lambda+A_{3} \quad, \quad G_{3}=A_{8} \Lambda^{2}+A_{g} \Lambda+A_{10} \quad$,

$G_{4}=A_{1} \Lambda^{3}+\Lambda^{4}+A_{2} \Lambda^{2}+A_{3} \Lambda+A_{4}$

$\operatorname{Re}\left[\frac{d \Lambda}{d \tau}\right]_{\Lambda=i \omega_{4}}^{-1}=\frac{P_{5 I} Q_{5 I}+P_{5 R} Q_{5 R}}{Q_{5 I}^{2}+Q_{5 R}^{2}}$,

Where

$$
\begin{aligned}
P_{5 I} & =\left(-A_{5} A_{8}^{4} \tau_{2}^{4}+\left(A_{10} A_{5} \tau_{2}+A_{6} A_{9} \tau_{2}+A_{7} A_{8} \tau_{2}\right.\right. \\
& \left.+A_{5} A_{9}-A_{6} A_{8}\right) \omega_{4}^{2}+A_{7} A_{9}-A_{10} A_{7} \tau_{2} \\
& \left.-A_{10} A_{6}\right) \cos \left(\omega \tau_{2}\right)+\left(\left(A_{5} A_{9} \tau_{2}+A_{6} A_{8} \tau_{2}\right) \omega_{4}^{3}\right. \\
& \left.+\left(2 A_{7} A_{8}-A_{10} A_{6} \tau_{2}-A_{7} A_{9} \tau_{2}-2 A_{10} A_{5}\right) \omega_{4}\right) \sin \left(\omega \tau_{2}\right) \\
& +\left(A_{1} A_{5}+3 A_{6}\right) \omega_{4}^{4}+\left(A_{3} A_{5}-3 A_{1} A_{7}-A_{2} A_{6}\right) \omega^{2}+A_{3} A_{7}-A_{4} A_{6},
\end{aligned}
$$

$P_{5 R}=\left(\left(-A_{5} A_{9} \tau_{2}-A_{6} A_{8} \tau_{2}\right) \omega_{4}^{3}+\left(A_{10} A_{6} \tau_{2}+A_{7} A_{9} \tau_{2}+2 A_{10} A_{5}\right.\right.$

$\left.\left.-2 A_{7} A_{8}\right) \omega_{4}\right) \cos \left(\omega_{4} \tau_{2}\right)+\left(A_{5} A_{8}^{A} \omega_{4}^{4} \tau_{2}+\left(A_{10} A_{5} \tau_{2}+A_{6} A_{9} \tau_{2}\right.\right.$

$\left.\left.+A_{7} A_{8} \tau_{2}+A_{5} A_{9}-A_{6} A_{8}\right) \omega_{4}^{2}+A_{7} A_{9}-A_{10} A_{7} \tau_{2}-A_{10} A_{6}\right) \sin \left(\omega_{4} \tau_{2}\right)$

$-2 A_{5} \omega_{4}^{5}+\left(2 A_{1} A_{6}+4 A_{7}\right) \omega_{4}^{3}+\left(2 A_{4} A_{5}-2 A_{2} A_{7}\right) \omega_{4}$, which lead to

$$
\begin{aligned}
Q_{5 I} & =\left(-A_{5} A_{8} \omega_{4}^{5}+\left(A_{10} A_{5}+A_{7} A_{8}\right) \omega_{4}^{3}-A_{10} A_{7} \omega_{4}\right) \cos \left(\omega_{4} \tau_{2}\right) \\
& +\left(A_{5} A_{9} \omega_{4}^{4}-A_{7} A_{9} \omega_{4}^{2}\right) \sin \left(\omega_{4} \tau_{2}\right)+A_{5} \omega_{4}^{7}+\left(-A_{2} A_{5}-A_{7}\right) \omega_{4}^{5} \\
& +\left(A_{2} A_{7}+A_{4} A_{5}\right) \omega_{4}^{3}-A_{4} A_{7} \omega_{4}, \\
Q_{5 R} & =\left(\left(-A_{5} A_{9}-A_{6} A_{8}\right) \omega_{4}^{4}+\left(A_{10} A_{6}+A_{7} A_{9}\right) \omega_{4}^{2}\right) \cos \left(\omega_{4} \tau_{2}\right) \\
& +\left(-A_{5} A_{8} \omega_{4}^{5}+\left(A_{10} A_{5}+A_{6} A_{9}+A_{7} A_{8}\right) \omega_{4}^{3}-A_{10} A_{7} \omega_{4}\right) \sin \left(\omega \tau_{2}\right) \\
& +\left(A_{1} A_{5}+A_{6}\right) \omega_{4}^{6}+\left(-A_{1} A_{7}-A_{2} A_{6}-A_{3} A_{5}\right) \omega_{4}^{4} \\
& +\left(A_{3} A_{7}+A_{4} A_{6}\right) \omega_{4}^{2} .
\end{aligned}
$$

Then, if

$P_{5 I} Q_{5 I}+P_{5 R} Q_{5 R}>0$,

holds, the root $\Lambda$ of (30) crosses the imaginary axis at $\tau_{1}=\tau_{4}^{c}$ from left to right.

\section{NUMERICAL EXPERIMENT}

In the numerical simulations, the following parameter values were used $\beta=3, \gamma=0.1, \mu=0.3, \eta=1, \omega=0.2$, $\lambda=0.005, \delta=0.2, \sigma=0.1, \xi=1, \kappa=0.05, \zeta=0.002, c=0.01$, $d=0.06, K=3500, Q=100, B=10$. These values were based on discussions with farmers and officials in Samut Songkram province.

Firstly we will shows the result when we apply the parameters to (9) which present the barn owl free system, the coconut yields become extinct as shown by Fig. 1 .
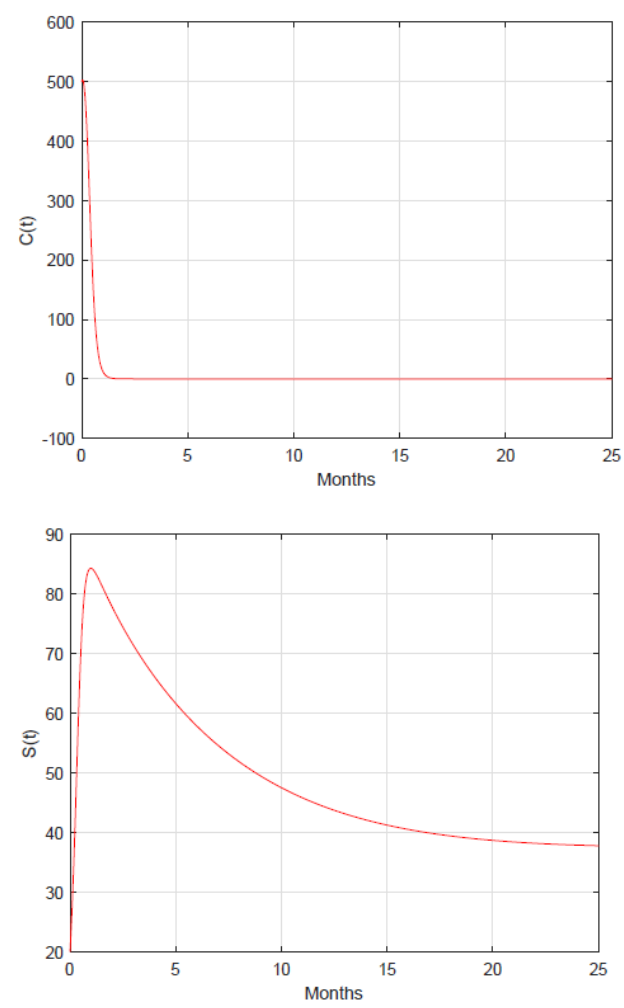

Fig. 1. Barn owls free system $\sigma=0.1$ with $C(0)=500$, $S(0))=20, O(0)=2, H(0)=2, \beta=3, \gamma=0.1, \mu=0.3, \eta=1$, $\omega=0.2, \lambda=0.005, \delta=0.2, \quad, \xi=1, \kappa=0.05, \zeta=0.002, c=0.01$, $d=0.06, K=3500, Q=100$, $B=10, \tau=0$. 
Since we need to keep coconuts positive, thus we needs to increase the predation rate of squirrel hunters, i.e., squirrel hunter must become more efficient in controlling squirrels. Suppose we raise this hunters'predation rate from $\sigma=0.1$ to $\sigma=0.8$, we have the results shown by Fig. 2 .
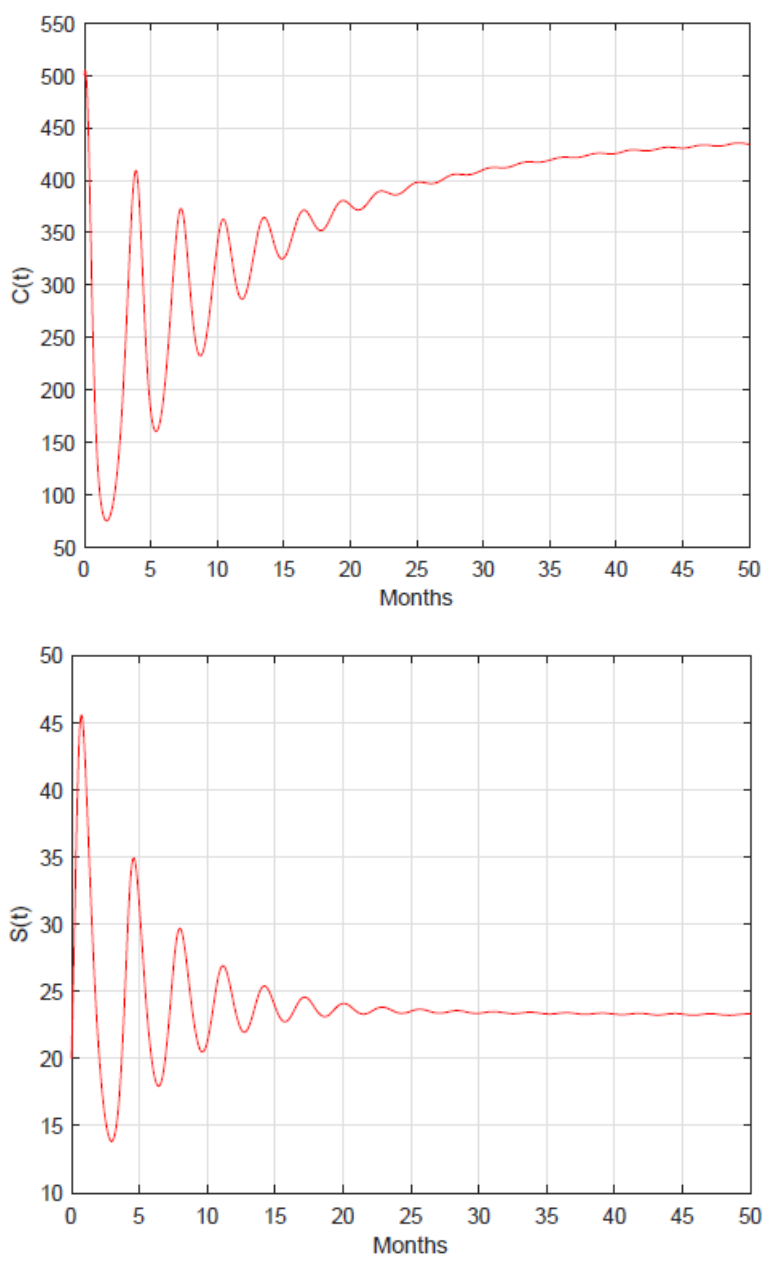

Fig. 2. Barn owls free system $\sigma=0.8$ with $C(0)=500$,

$S(0))=20, O(0)=2, H(0)=2, \beta=3, \gamma=0.1, \mu=0.3, \eta=1$, $\omega=0.2, \lambda=0.005, \delta=0.2, \quad \xi=1, \kappa=0.05, \zeta=0.002, c=0.01$, $d=0.06, K=3500, Q=100, B=10, \tau=0$.

The effect of time delay $\tau$ can be investigated by assuming that squirrel prefer to eat the coconuts at particular age $\tau$. The variation of this $\tau$, however, can change the solutions of (9), i.e., it produces different effects when its values are below or above the critical value. In this case, the critical value $\tau_{c 0}=0.096$ calculated from $\omega=2.319$. According to critical value of $\tau$, it indicates that when $\tau \in[0,0.096), E_{8_{0}}=(443,23,4)$ became asymptotically stable.

However when $\tau=0.095<0.096$, the waveforms and the phase plots of system (9) were changed which shown by Fig. 3. When $\tau=0.097>0.096, E_{8_{0}}$ became bifurcated as show by

Fig. 4.
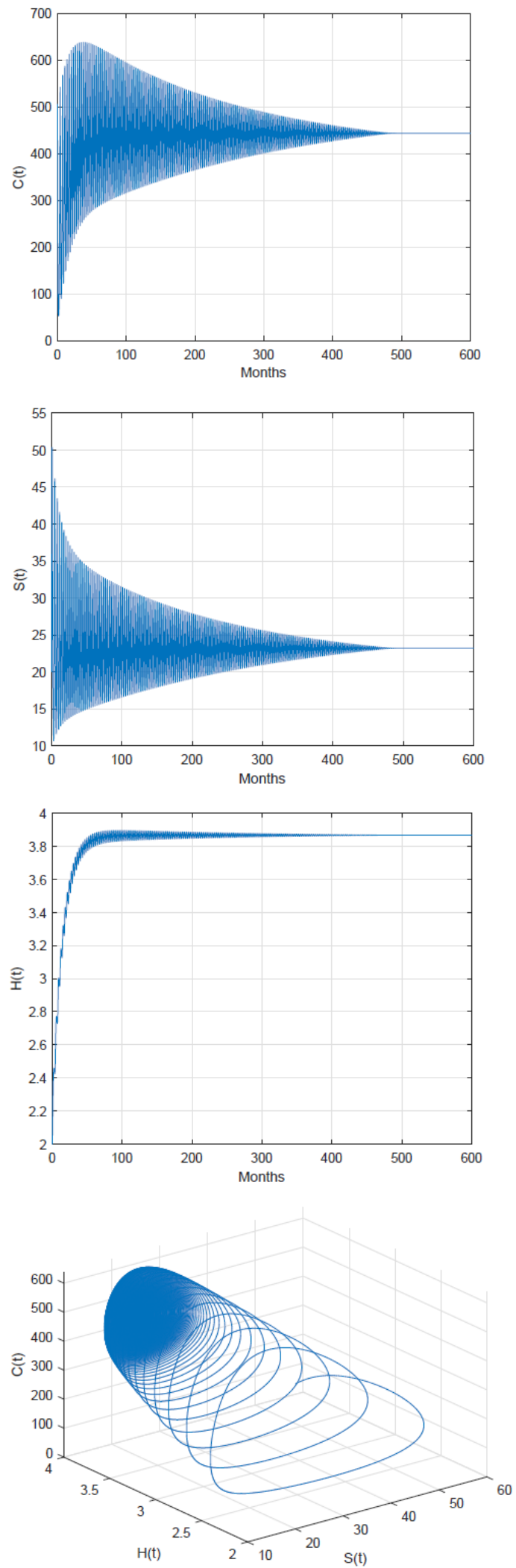

Fig. 3. $\mathrm{CSOH}$ system with $C(0)=500, S(0))=20, O(0)=2$, $H(0)=2, \beta=3, \gamma=0.1, \mu=0.3, \eta=1, \omega=0.2, \lambda=0.005$, $\delta=0.2, \quad \sigma=0.8, \xi=1, \kappa=0.05, \zeta=0.002, c=0.01, d=0.06$, $K=3500, Q=100, B=10, \tau=0.095$. 

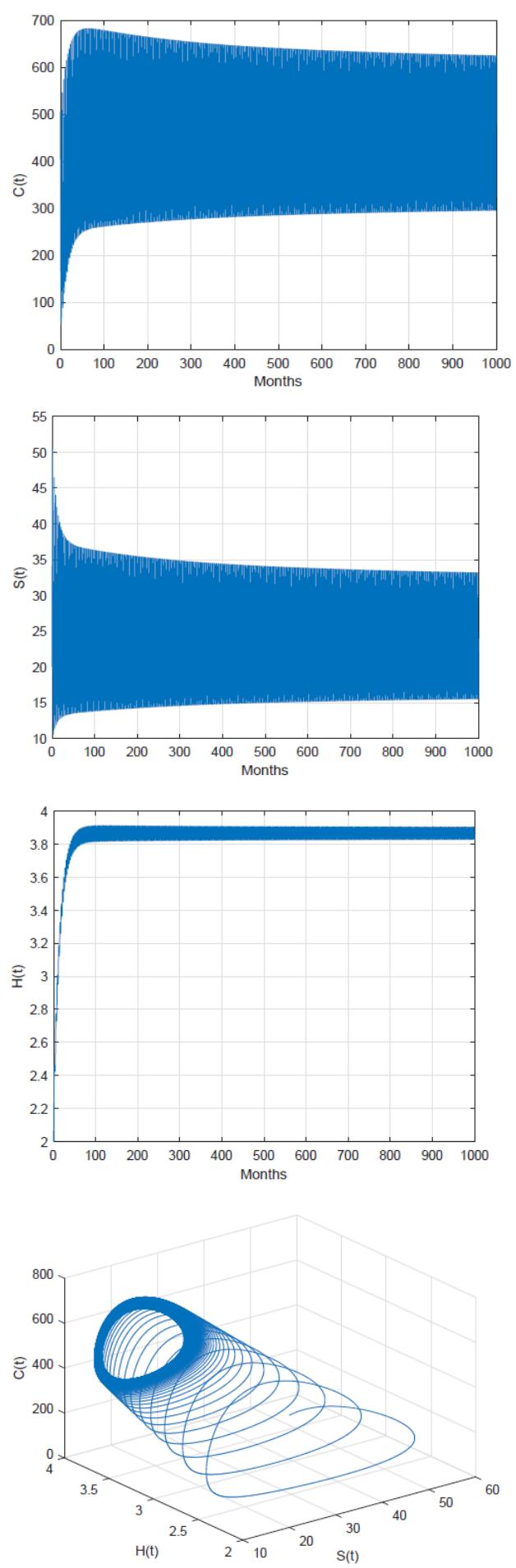

Fig. 4. $\mathrm{CSOH}$ system with $C(0)=500, S(0))=20, O(0)=2$, $H(0)=2, \beta=3, \gamma=0.1, \mu=0.3, \eta=1, \omega=0.2, \quad \lambda=0.005$, $\delta=0.2, \quad \sigma=0.8, \xi=1, \kappa=0.05, \zeta=0.002, c=0.01, d=0.06$, $K=3500, Q=100, B=10, \tau=0.097$.

Now we consider the time delay system (1). Although this system have two time delays, i.e., $\tau_{1}$ and $\tau_{2}$, only $\tau_{1}$ can cause the bifurcation in $E_{8}=(375,23,11,4)$. This can observe from the values of the $\omega$ of $\tau_{2}$ which provide no real number but $\pm 2.202187149 \pm 0.2442798467 I, \pm 0.06132045903 I$, $\pm 1.013195741 \mathrm{I}$, and $\pm 2.202187149 \pm 0.2442798467 I$. For $\tau_{1}$, there are $\omega$ value of \pm 2.416 which signal the occurrence of the bifurcation. By using the positive value of $\omega$, the critical value is given by $\tau_{c 0}=0.114$. According to critical value of $\tau_{1}$, it indicate that when $\tau_{1} \in[0,0.114), E_{8}=(375,23,11,4)$ became asymptotically stable. By setting $\tau_{1}=0.113<0.114$, the waveforms and the phase plots of system (1) were shown in Fig. 5. When $\tau_{1}=0.115>0.114$, it could be observed that $E_{14}=(375,23,11,4)$ lost its stability and became bifurcated as show by Fig. 6.
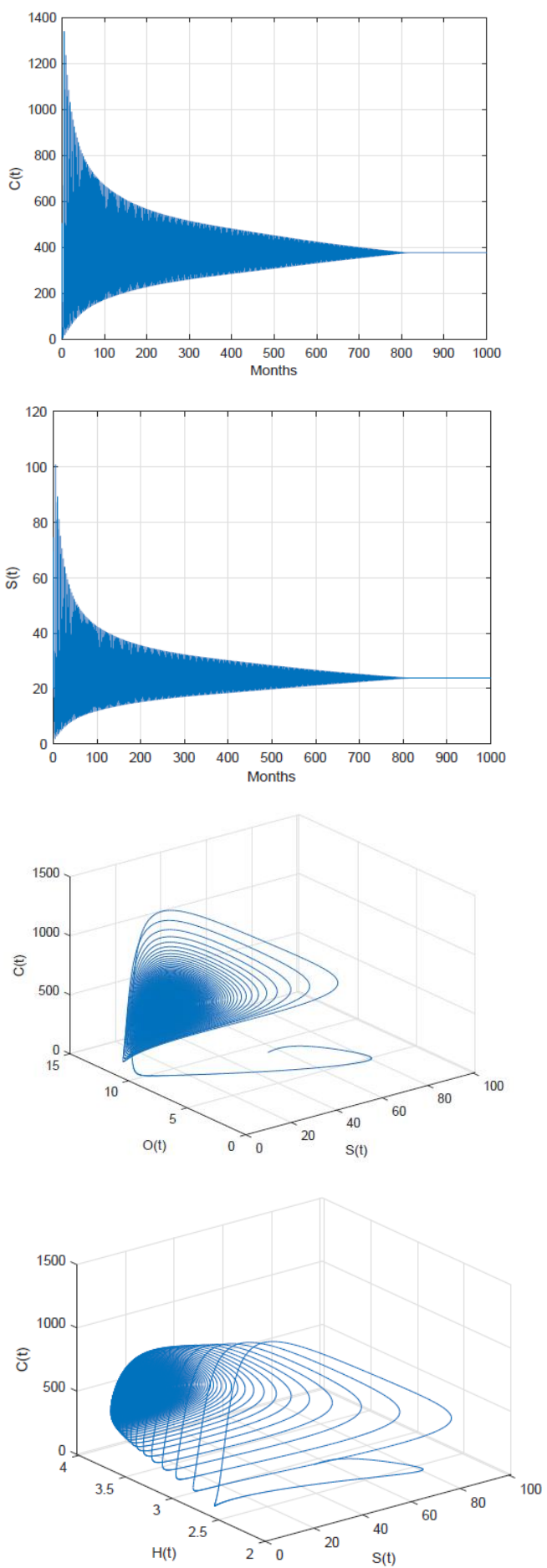

Fig. 5. $\mathrm{CSOH}$ system with $C(0)=500, S(0))=20, O(0)=2$, $H(0)=2, \beta=3, \gamma=0.1, \mu=0.3, \eta=1, \omega=0.2, \lambda=0.005$, $\delta=0.2, \sigma=0.8, \xi=1, \kappa=0.05, \zeta=0.002, c=0.01, d=0.06$, $K=3500, Q=100, B=10, \tau_{1}=0.113$, and $\tau_{2}=0$.

\section{Published By:}



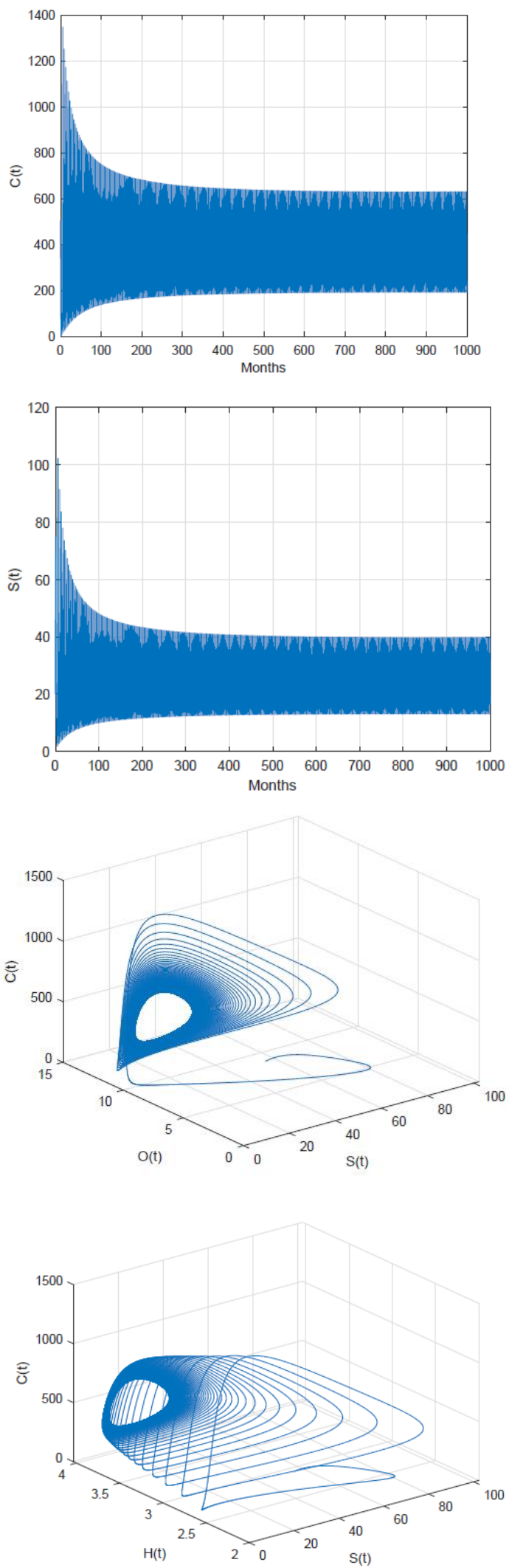

Fig. 6. $\mathrm{CSOH}$ system with $C(0)=500, S(0))=20, O(0)=2$, $H(0)=2, \beta=3, \gamma=0.1, \mu=0.3, \eta=1, \omega=0.2, \lambda=0.005$, $\delta=0.2, \sigma=0.8, \xi=1, \kappa=0.05, \zeta=0.002, c=0.01, d=0.06$, $K=3500, Q=100, B=10, \tau_{1}=0.115$, and $\tau_{2}=0$.

The effect of time delay can be extended by increasing the time delay. In this manner, the squirrel newborn should be slow down, and the coconut yield should be increased. By setting $\tau_{1}=0.2>0.114$, the waveforms and the phase plots of system (1) were represented by Fig. 7 which shown a large oscillation of all species.
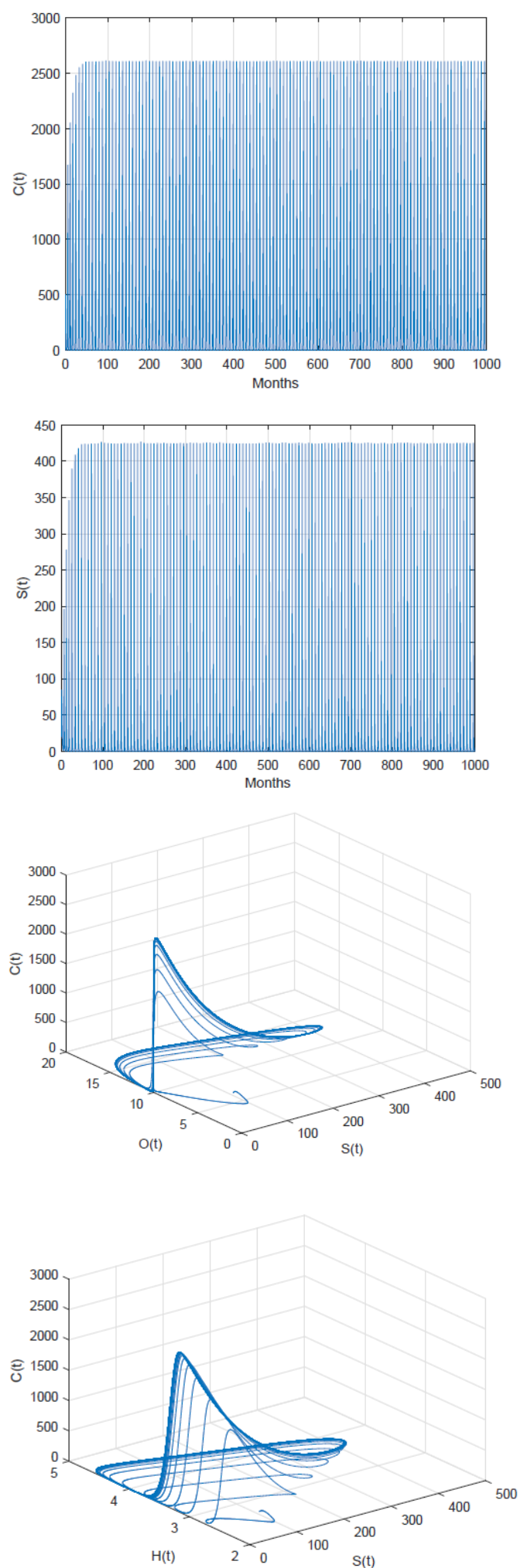

Fig. 7. $\mathrm{CSOH}$ system with $C(0)=500, S(0))=20, O(0)=2$, $H(0)=2, \beta=3, \gamma=0.1, \mu=0.3, \eta=1, \omega=0.2, \lambda=0.005$, $\delta=0.2, \quad \sigma=0.8, \xi=1, \kappa=0.05, \zeta=0.002, c=0.01, d=0.06$, $K=3500, Q=100, B=10, \tau_{1}=0.2$, and $\tau_{2}=0$.

\section{Published By:}


To suppress the fluctuation of coconut yields to meet the objective of a stable income of farmers, we suggest two possible strategies. The first strategy is to reduce the squirrel coconut eating rate $\gamma$. By changing $\gamma=0.1$ to $\gamma=0.08$, all solution of (1) become asymptotically stable as shown by Fig. 8. This strategy can be implemented by searching for methods to protect coconut yields from squirrels. The traditional method that the farmers used for this purpose is to cover some part of the coconut tree to make difficulty for squirrel climbing. However, only this method may be less effective since squirrels can jump between the coconut trees. Therefore the range between the coconut trees should be designed properly to avoid squirrels do their jump. The chemical spray on coconut yields may be a possible alternative to protect coconut from squirrel eating them. Nevertheless, the farmers have to recognize the negative side effect of this practice. The second strategy is to reduce the new squirrel conversion rate $\lambda$. The results of changing $\lambda$ from $\lambda=0.005$ to $\lambda=0.004$ were shown by Fig. 9. To implement this strategy, the farmers may utilize the chemical substance that can help to slow down this rate. However, this chemical substance is not known or even recognized by the farmers. Thus the first strategy may be more practical when compared to the second one.
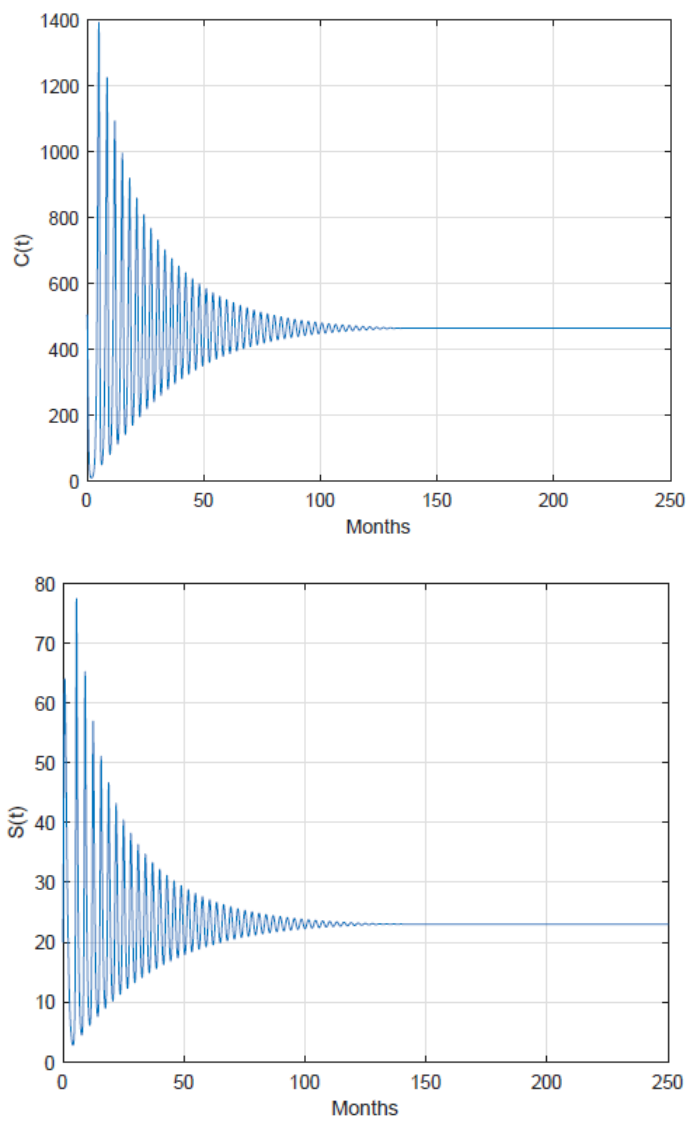

Fig. 8. Policy 1: $\gamma=0.08$ with $C(0)=500, S(0))=20, O(0)=2$, $H(0)=2, \beta=3, \mu=0.3, \eta=1, \omega=0.2, \lambda=0.005, \delta=0.2$, $\sigma=0.8, \xi=1, \kappa=0.05, \zeta=0.002, c=0.01, d=0.06, K=3500$, $Q=100, B=10, \tau_{1}=0.115$, and $\tau_{2}=0$.
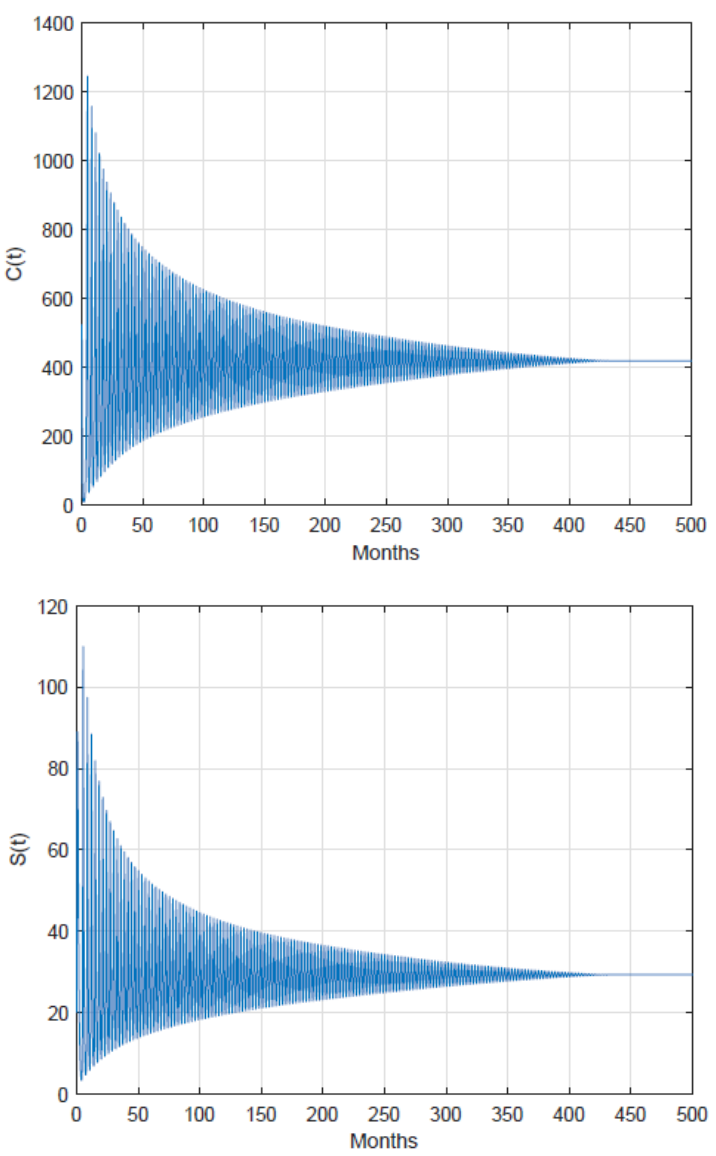

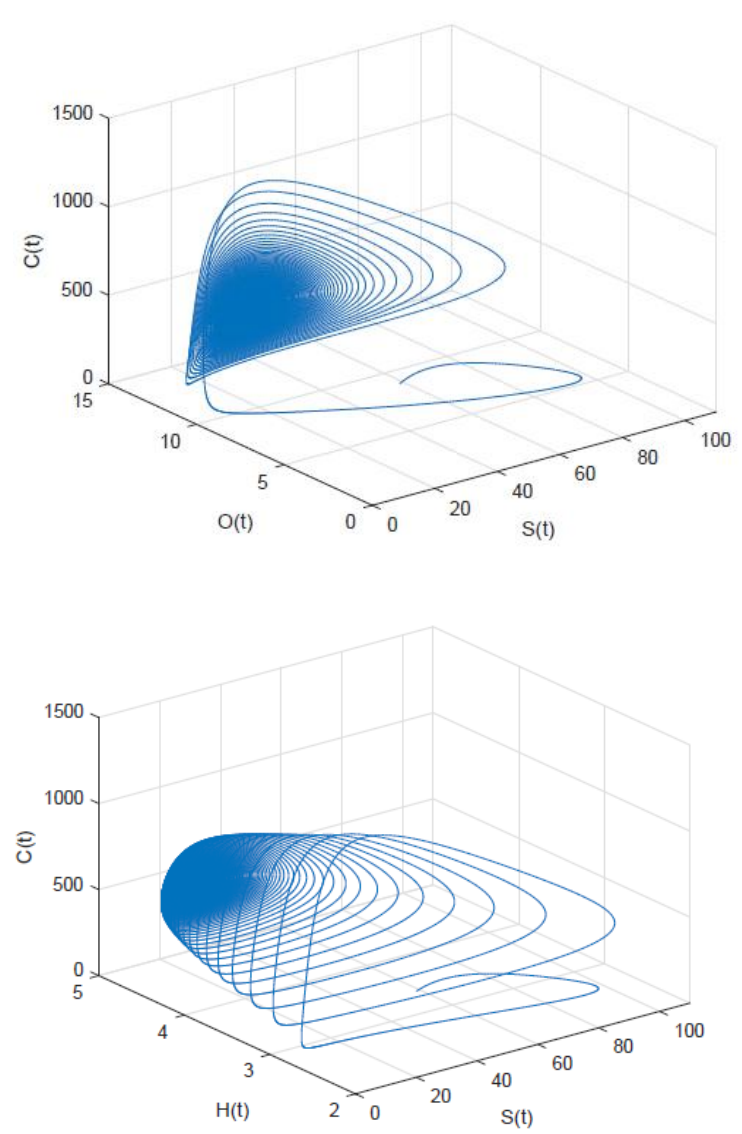

Fig. 9. Policy $2 \lambda=0.004$ with $C(0)=500, S(0))=20$, $O(0)=2, H(0)=2, \beta=3, \gamma=0.1, \mu=0.3, \eta=1, \omega=0.2$, $\delta=0.2, \sigma=0.8, \xi=1, \kappa=0.05, \zeta=0.002, c=0.01, d=0.06$, $K=3500, Q=100, B=10, \tau_{1}=0.115$, and $\tau_{2}=0$.

\section{Conclusions}

In this paper, we propose the delay $\mathrm{CSOH}$ model to explain the dynamics on the coconut farm. We investigate the time delay effect on the dynamics of coconut yields, squirrels, barn owls, and squirrel hunters. The results of the analysis showed that time delay are a cause of Bifurcation that makes the number of coconut yields unstable. Therefore to stabilize the coconut yields which imply income stability of farmers, we suggest two possible strategies which can lead the farmers to meet this stability objective.

\section{ACKNOWLEDGMENT}

This research is supported by the Centre of Excellence in Mathematics, the Commission on Higher Education, Thailand.This work is also supported by the Department of Mathematics, King Mongkut's University of Technology North Bangkok, Thailand.

\section{REFERENCES}

1. A. A. Berryman, "The orgins and evolution of predator-prey theory," Ecology, vol. 73, no. 5, 1992, pp. 1530-1535.

2. B. Sahoo, "Predator-prey model with different growth rates and different functional responses: a comparative study with additional food," Int. J. Appl. Math. Res., vol. 1, no. 2, 2012, pp. 117-129.

3. M. Javidi, "NematNyamorady, Allee effects in a predator prey system with a saturated recovery function and harvesting," Int. J. Adv. Math. Sci., vol. 1 , no. 2, 2013, pp. 33-44.
4. O. Misra, and A. Babu, "A model for the effect of toxicant on a three species food-chain system with food-limited growth of prey population,' Glob. J Math Anal, vol. 2, no. 3, 2014, pp. 120-145.

5. A. A. Majeed, and R. R. Saadi, "The persistence of prey-predator model with competition hosts," Int. J. Appl. Math. Res., vol. 4, no. 2, 2015, pp 351-361.

6. R. D. Amalia, D. K. Arif, and others, "Optimal control of predator-prey mathematical model with infection and harvesting on prey," in Journal of Physics: Conference Series, vol. 974, 2018, pp. 012050.

7. M. Agarwal, and P. Pandey, "A stage-structured predator-prey model with disease in the prey," in Journal of Physics: Conference Series, 2008, vol. 96, p. 012118.

8. J. Yan-Fei, and X. Wen-Xian, "Stability of a delayed predator-prey model in a random environment," Chin. Phys. B, vol. 24, no. 11, 2015, p 110501.

9. R. Sivasamy, S. Vinoth, and K. Sathiyanathan, "Exploring the dynamics of three species delayed food chain model with harvesting," presented at the Journal of Physics: Conference Series, vol. 1139, 2018, p. 012028.

10. Z. Zhang, H. Yang, and M. Fu, "Hopf bifurcation in a predator-prey system with Holling type III functional response and time delays," J. Appl. Math. Comput., vol. 44, no. 1-2, 2014, pp. 337-356.

11. C. Xu, and P. Li, "Dynamical analysis in a delayed predator-prey model with two delays," Discrete Dyn. Nat. Soc., vol. 2012, 2012, p. 22.

12. Q. Liu, Y. Lin, and J. Cao, "Global Hopf bifurcation on two-delays Leslie-Gower predator-prey system with a prey refuge," Comput. Math Methods Med., vol. 2014, 2014, p. 12.

13. Z. Zhang and Y. Wang, "SVEIRS epidemic model with delays and partial immunization for internet worms," J. Appl. Math. Comput., vol. 57, no. 1-2, 2018, pp. 333-358.

14. M. Peng, and Z. Zhang, "Hopf bifurcation analysis in a predator-prey model with two time delays and stage structure for the prey," Adv. Differ. Equ., vol. 2018, no. 1, 2018, p. 251.

15. P. Feng, "Analysis of a delayed predator-prey model with ratio-dependent functional response and quadratic harvesting," J. Appl. Math. Comput., vol. 44, no. 1-2,2014, pp. 251-262.

16. S. Kundu, and S. Maitra, "Permanence And Global Stability Analysis Of A Delayed Three Species Predator-Prey Model With Cooperation Among Preys," vol. 30, no. 1, 2018, pp. 11-26.

17. R. N. Shalan, "Local Stability of Prey-Predator with Holling type IV Functional Response," Glob. J. Pure Appl. Math., vol. 13, no. 3, 2017, pp. 967-980.

\section{Authors Profile}

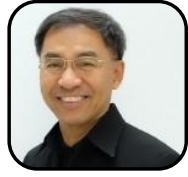

Sanoe Koonprasert received B.Ed. in Mathematics and M.Ed. in Mathematics from Srinakharinwirot University Prasanmit Thailand. He obtained M.S. and Ph.D. in Mathematics from Montana State University, USA. He has worked in Department of Mathematics, Faculty of Applied Science, King Mongkut's University of Technology North Bangkok, Thailand where he is currently an Associate Professor and he is also a researcher in the Centre of Excellence in Mathematics, CHE, Si Ayutthaya Road, Bangkok, Thailand. His research interests include Linear - Non Linear, ODE/PDE system, Finite Element Methods, PDE Methods, Numerical ODE/PDE, Mathematical Model, and Dynamical System. He has published many research papers in various journals and conferences.

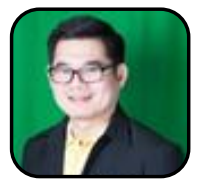

Sekson Sirisubtawee received B.Eng. in Electronics Engineering and M.S. in Applied Mathematics from King Mongkut's Institute of Technology Ladkabang, Thailand. He obtained M.S. and Ph.D. in Applied Mathematics from University of Colorado at Boulder, USA. He has worked in the department of Mathematics, faculty of Applied Science, King Mongkut's University of Technology North Bangkok, Thailand. $\mathrm{He}$ is currently an assistant professor there and he is also a researcher in the Centre of Excellence in Mathematics, CHE, Si Ayutthaya Road, Bangkok, Thailand. His research interests include numerical analysis, differential equations, mathematical modeling, exact solutions of PDEs. He has published many research papers in various journals and conferences. 
Adirek Vajrapatkul received B.Econ. from Sukhothai Thammathirat Open University, Thailand and M.Econ. from National Institute of Development Administration, Thailand. He is currently a Ph.D. in Applied Mathematics student in the Department of Mathematics, Faculty of Applied Science, King Mongkut's University of Technology North Bangkok, Thailand. He has worked in Faculty of Management Science, Bansomdejchaopraya Rajabhat University, Thailand. His research interests include Linear - Non Linear, ODE system, Numerical ODE, Mathematical Model, and Dynamical System. He has presented several research papers in National and International conferences and published research papers in journals. 